\title{
A general equilibrium evolutionary model with two groups of agents, generating fashion cycle dynamics
}

\author{
Ahmad Naimzada • Marina Pireddu
}

Received: date / Accepted: date

\begin{abstract}
We propose a discrete-time exchange economy evolutionary model with two groups of agents. In our setting the definition of equilibrium depends also on agents' population shares, which affect the market clearing conditions. We prove that, despite such difference with the classical Walrasian framework, for all economies and population shares there exists at least one equilibrium and we show that, for all population shares, generically in the set of the economies, equilibria are finite and regular. We then introduce the dynamic law governing the evolution of the population shares, and we investigate the existence and the stability of the resulting stationary equilibria. We assume that the reproduction level of a group is related to its attractiveness degree, which depends on the social visibility level, determined by the consumption choices of the agents in that group. The attractiveness of a group is described via a generic bell-shaped map, increasing for low visibility levels, but decreasing when the visibility of the group exceeds a given threshold value, due to a congestion effect. The model is able to reproduce the recurrent dynamic behavior typical of the fashion cycle, presenting booms and busts in the agents' consumption choices, and in the groups' attractiveness and population shares.
\end{abstract}

Keywords General equilibrium · Evolutionary mechanism · Social interaction · Fashion cycle · Bifurcation · Multistability

JEL Classification C62 - D11 · D51 · D91

A. Naimzada

University of Milano-Bicocca, Dept. of Economics, Management and Statistics,

U6 Building, Piazza dell'Ateneo Nuovo 1, 20126 Milano, Italy.

Tel.: +390264485813

E-mail: ahmad.naimzada@unimib.it

M. Pireddu (corresponding author)

University of Milano-Bicocca, Dept. of Mathematics and its Applications,

U5 Building, Via R. Cozzi 55, 20125 Milano, Italy.

Tel.: +390264485767

E-mail: marina.pireddu@unimib.it 


\section{Introduction}

In the present paper we propose a discrete-time exchange economy evolutionary model in view of providing a formal representation of the fashion cycle, i.e., the oscillatory behavior of the variable describing the consumed or purchased amount of a certain good, characterized by booms and busts.

Although in the mainstream economic theory of consumption it is usually supposed that preferences are exogenously fixed, two research strands removed such modeling assumption. While in the former (see e.g. Day 1970; Benhabib and Day 1981) the endogenous change in preferences is described as a consequence of the past choices made by the consumer itself, in the latter the change in preferences is due to social interaction with other agents, in agreement with Veblen (1899), Simmel (1904) and Leibenstein (1950). The second, more multidisciplinary, viewpoint has led to many economic contributions, such as the works by Gaertner (1974, 1987), by Gaertner and Jungeilges $(1988,1993,1994)$ and by Ekaterinchuk et al. (2017, 2018).

In particular, Simmel (1904) argues that the fashion cycle is driven by two opposite forces: imitation and distinction. Following such idea, thanks to our evolutive general equilibrium setting we may view the fashion cycle as the combined result of three ingredients: the price formation mechanism, the population share updating rule, and the social interaction of two groups of agents exhibiting both bandwagon and snob behaviors. Namely, according to Vigneron and Johnson (1999), on the basis of the empirical literature, too, in the context, e.g., of luxury and prestige-seeking consumption agents may oscillate between imitative and snob attitudes.

Looking more closely at the three building blocks of our model, each of them plays an important role. The general equilibrium approach allows us to give a rigorous microfoundation to the agents' consumption activity and to the price formation mechanism. An updating rule is needed in order to investigate the evolution of agents' shares over time. Finally, the bandwagon and snob behaviors arise from an analytical description of the social interaction process through bell-shaped attractiveness functions associated to the different lifestyles.

In the past decades, some models have been proposed to give a formal representation of the fashion cycle, in which one of the three above mentioned ingredients was privileged. ${ }^{1}$ More precisely, in Frijters (1998) just the price formation mechanism operates and the fashion cycle may occur according to the relative values of the current and of the future price. Matsuyama (1991), in a continuous-time setting, and Gardini et al. (2018), in a discrete-time framework involving discontinuous piecewise linear maps, deal with evolutive aspects, only. Finally, Caulkins et al. (2007), Coelho and McClure (1993), Corneo and Jeanne (1999), Di Giovinazzo and Naimzada (2015), Karni and Schmeidler (1990), Pesendorfer (1995) and Zhang (2016, 2017) propose mod-

\footnotetext{
1 Oscillatory dynamics in the consumption activities are found, in different contexts, also in Antoci et al. (2004), Bischi and Radi (2012), Matsumoto (2003) and Naimzada et al. (2013).
} 
els in which the fashion cycle originates from some form of social interaction occurring between consumers or firms.

On the other hand, we aim to give a comprehensive description of the fashion cycle. In more detail, our approach is based on some of the modeling choices made in Chang and Stauber (2009) and in Naimzada and Pireddu (2016), where continuous-time evolutive general equilibrium models were proposed. However, in those contributions, agents' preferences were represented via Cobb-Douglas utility functions only, and the share updating mechanism was based on biological aspects concerning the calorie intakes by the agents belonging to the two groups. Moreover, we recall that in Chang and Stauber (2009) and in Naimzada and Pireddu (2016) just trivial dynamic behaviors could arise. Hence, such frameworks were not suitable to describe the fashion cycle, neither for their modeling features, nor for the resulting dynamic outcomes. In fact, the two papers that bear more resemblance to the setting we present are Naimzada and Pireddu $(2018,2019 a)$, where, starting from Chang and Stauber (2009) and Naimzada and Pireddu (2016), we dealt with a share updating rule based on sociological aspects. In more detail, the evolutionary mechanism was described via the discrete exponential replicator rule (see e.g. Branch and McGough 2008; Cabrales and Sobel 1992; Hofbauer and Weibull 1996; Nachbar 1990; Sandholm 2010; Taylor and Jonker 1978), in which the reproduction level of a group is based on a comparison between the attractiveness degree of the two groups of agents. The attractiveness of a group depends on its social visibility level, which is determined as a linear combination of the amount of the two goods consumed in equilibrium by the agents in that group, multiplied by commodity-specific visibility factors. Such social parameters measure the capability of consumption to convey ideas and transmit information about the status of the agents who consume that commodity (see Heffetz 2011, 2012 for an explanation about how those parameters can be estimated). The attractiveness of a group is initially increasing with its visibility level, and this describes the bandwagon regime, characterized by an imitative behavior. However, when the visibility of a group exceeds a given threshold value, a congestion effect is produced. The attractiveness of that group then becomes a decreasing function of its visibility level, and this describes the snob regime, characterized by a predominating wish for distinction. While in Naimzada and Pireddu (2018) just one particular formulation for the attractiveness was considered, in Naimzada and Pireddu (2019a) generic bell-shaped attractiveness functions for the two groups of agents were taken into account. The functioning of both frameworks, which can generate interesting dynamics and also the fashion cycle, is described in what follows. As mentioned above, the share updating mechanism is based on a comparison between the attractiveness of the two groups of agents. In such process, the social interaction affects the visibility levels, that are also influenced by the commodity-specific visibility factors. The evolutive rule determines the new population shares, whose values in turn affect the equilibrium price and the equilibrium consumption choices. The latter quantities enter the visibility expression, which is involved in the share updating rule, and the process repeats again. 
The main weakness in Naimzada and Pireddu (2018, 2019a) lies in the fact that agents' preferences were described by Cobb-Douglas utility functions only. In order to fix such issue, in the present contribution we keep the generality concerning the formulation of the attractiveness functions achieved in Naimzada and Pireddu (2019a), adding to this an increased generality in regard to the description of agents' preferences. Namely, we here suppose that agents' utility functions are free to vary in a suitable set of maps. Such new assumption urges us to investigate not only the dynamic features of our model, but also to study the existence and the generic regularity of the market equilibria, which link the equilibrium price and the optimal consumption choices to population shares. Namely, due to the presence of the agents' population shares inside the market clearing conditions, the validity of the standard results about exchange economies has to be carefully verified. We perform such task by adopting the extended approach illustrated in Villanacci et al. (2002). More precisely, using the homotopy invariance property of the topological degree (see Lloyd 1978 for this and related topological degree results) ${ }^{2}$ we show that for all economies and population shares there exists at least a market equilibrium and, employing a tranvsersality argument, we prove that, for all population shares, generically in the set of the economies, equilibria are finite and regular. In particular, due to the need of dealing with function spaces, we have to employ the infinite-dimensional version of the Implicit Function Theorem by Gloeckner (2006), used in the restricted participation frameworks in Carosi et al. (2009), Gori et al. (2013) and Hoelle et al. (2015), too. Furthermore, in order to avoid indeterminacy issues, we check that a unique equilibrium exists when considering utility functions that yield individual demand functions with the gross substitute property. We then introduce the concept of market stationary equilibrium, that is a market equilibrium which does not vary with time and that solves in any period the dynamic equation describing the share updating rule, where the latter, as explained above, maintains the generality reached in Naimzada and Pireddu (2019a), encompassing generic bell-shaped attractiveness functions for the two groups of agents. After deriving the expression of the market stationary equilibria, we investigate their local stability.

In order to illustrate the general results that we obtain on existence and local stability of the stationary equilibria, as well as to analyze the emergence of fashion cycle dynamics, we add a simulative section where we focus on StoneGeary utility functions (see Geary 1950 and Stone 1954), which generalize the Cobb-Douglas utility functions, and where we consider the formulation of the attractiveness map dealt with in Naimzada and Pireddu (2018). ${ }^{3}$ Performing a bifurcation analysis on varying the parameter representing a suitable sensitivity measure, we find that the nontrivial equilibrium, characterized by the coexistence between the agents of the two groups, loses stability via a

\footnotetext{
2 Cf. also Gori et al. (2014), where an homotopy method is employed to prove existence of equilibria in a model with real assets and restricted participation.

3 We refer the reader to Naimzada and Pireddu (2019b) for a more detailed bifurcation analysis, conducted for several parameter configurations and for the formulations of the attractiveness maps introduced in Naimzada and Pireddu (2019a), too.
} 
flip bifurcation in favor of a stable period-two cycle. Like in Naimzada and Pireddu $(2018,2019$ a), also in this work we detect interesting multistability phenomena, involving equilibria, as well as periodic or chaotic attractors, and oscillatory behaviors both for the population shares and the consumed quantities are allowed. In particular, the dynamic coexistence between groups and the oscillatory nature of the consumption activities display the recurrent dynamic behavior typical of the fashion cycle, with booms and busts. In view of better understanding the functioning of the model dynamics, we also provide an interpretation of the main scenarios we found in our bifurcation analysis from a sociological and economic viewpoint, in terms of visibility and attractiveness.

The remainder of the paper is organized as follows. In Section 2 we present and study our model. In particular in Subsection 2.1 we analyze the existence, generic regularity and uniqueness of Walrasian equilibria with agents' population shares under suitable conditions on the economies, while in Subsection 2.2 we illustrate the dynamic equation describing the share updating rule and, after introducing the concept of stationary equilibria, we investigate their existence and local stability. In Section 3 we show the emergence of fashion cycle dynamics for our system when considering Stone-Geary utility functions, providing a sociological and economic interpretation of the main scenarios we find. In Section 4 we briefly discuss our results and describe some variants and extensions of the model. The Appendix contains the proofs.

\section{The model}

\subsection{The Walrasian equilibria with shares}

Let us consider an exchange economy with a continuum of agents, which may be of type $\alpha$ or of type $\beta .{ }^{4}$ There are two consumption goods, $x$ and $y$, and agents' preferences, as in most literature on smooth economies (see e.g. Villanacci et al. 2002), are described by the class of utility functions introduced in the following definition.

Definition 1 For $i \in\{\alpha, \beta\}$, we define $\mathcal{U}_{i}$ as the set of utility functions $u_{i}:(0,+\infty)^{2} \rightarrow \mathbb{R}$ such that

(A1) $u_{i} \in \mathcal{C}^{2}\left((0,+\infty)^{2}\right)$;

(A2) $u_{i}$ is differentiably strictly increasing, i.e., $D u_{i}(x, y)>>0, \forall(x, y) \in(0,+\infty)^{2}$

(A3) $u_{i}$ is differentiably strictly quasiconcave, i.e., $D u_{i}(x, y) v=0$ implies $v D^{2} u_{i}(x, y) v<0, \forall(x, y) \in(0,+\infty)^{2}, v \in \mathbb{R}^{2} \backslash\{(0,0)\}$

(A4) $\forall(\bar{x}, \bar{y}) \in(0,+\infty)^{2},\left\{(x, y) \in(0,+\infty)^{2}: u_{i}(x, y) \geq u_{i}(\bar{x}, \bar{y})\right\}$ is closed in the topology of $\mathbb{R}^{2}$.

\footnotetext{
4 We keep the notation used in Chang and Stauber (2009) and in Naimzada and Pireddu (2016, 2018, 2019a), where $i \in\{\alpha, \beta\}$ was the weight assigned to good $x$ in the Cobb-Douglas utility functions by agents in group $i$, with $0<\alpha<\beta<1$.
} 
Assumption $(A 1)$ allows to perform computations and to employ tools from Calculus such as the implicit function theorem. Assumption $(A 2)$ says that households always prefer a bundle with slightly more of anything, no matter what they are consuming. Assumption $(A 3)$ says that households prefer bundles in which commodities are fairly distributed and ensures the existence of a unique solution to the household maximization problem. Assumption (A4) implies that indifference curves of utility functions do not touch the axes and thus that the solution is interior. By the implicit function theorem, it is possible to show that such solution depends in a smooth manner on endowments and on prices (see Theorem 8.3.397 in Villanacci et al. 2002). Actually, employing an extension of the implicit function theorem valid for maps from topological vector spaces to Banach spaces by Gloeckner (2006) (see Theorem 2 below), it can be easily shown that the solution to the household maximization problem depends in a smooth manner also on utility functions.

We notice that, by Definition 1 , we have $\mathcal{U}_{\alpha}=\mathcal{U}_{\beta}$. Hence, we will denote the set of utility functions simply by $\mathcal{U}$.

In our model we assume that time is discrete, i.e., that $t \in \mathbb{N}$. The quantity of good $x(y)$ consumed by an agent of type $i \in\{\alpha, \beta\}$ at time $t$ is denoted by $x_{i, t}\left(y_{i, t}\right)$. Both kinds of agents have the same positive endowments of the two goods, denoted respectively by $w_{x}$ and $w_{y}$. We define the set of economies as $\mathcal{E}=\mathcal{U}^{2} \times(0,+\infty)^{2}$, containing the elements $E=\left(u_{\alpha}, u_{\beta}, w_{x}, w_{y}\right) .{ }^{5}$ In view of the result on generic regularity of market equilibria (cf. Proposition 2), we assume that $\mathcal{E} \subseteq \mathscr{E}$ is endowed with the topology induced by the Hausdorff topological vector space

$$
\mathscr{E}=\left[\mathcal{C}^{2}\left((0,+\infty)^{2}\right)\right]^{2} \times \mathbb{R}^{2}
$$

where $\mathscr{E}$ is endowed with the product topology of the natural topologies on each of the spaces in the Cartesian product. In particular, on the $\mathcal{C}^{2}$ function space we consider the $\mathcal{C}^{2}$ compact-open topology.

We denote by $p_{x, t}>0$ and $p_{y, t}>0$ the prices at time $t$ for goods $x$ and $y$, respectively. The size of the population of kind $\alpha(\beta)$ at time $t$ is denoted by $A_{t}\left(B_{t}\right)$. The normalized variable $a_{t}=A_{t} /\left(A_{t}+B_{t}\right) \in[0,1]$ represents the population fraction composed by the agents of type $\alpha$ and $b_{t}=1-a_{t}=$ $B_{t} /\left(A_{t}+B_{t}\right) \in[0,1]$ represents the population fraction composed by the agents of type $\beta$.

We are now ready to provide the definition of market equilibrium.

Definition 2 Given the economy $E \in \mathcal{E}$ and the population share $a_{t} \in[0,1]$, a market equilibrium at time $t$ is a vector $\left(p_{x, t}^{*}, p_{y, t}^{*}, x_{i, t}^{*}, y_{i, t}^{*}\right)$, with $i \in\{\alpha, \beta\}$, such that:

\footnotetext{
5 We notice that if $u_{\alpha}=u_{\beta}$ then agents are homogeneous since endowments of both goods coincide between groups. In order not to overburden notation and not to excessively complicate the analysis, we will here focus on the case with $w_{x, \alpha}=w_{x, \beta}=w_{x}$ and $w_{y, \alpha}=$ $w_{y, \beta}=w_{y}$, implicitly assuming that $u_{\alpha} \neq u_{\beta}$, like it happens in the example considered in Section 3.
} 
- every kind of agent $i$ chooses a utility-maximizing consumption bundle $\left(x_{i, t}^{*}, y_{i, t}^{*}\right)$, given $\left(p_{x, t}^{*}, p_{y, t}^{*}\right)$, i.e., the agents of group $i \in\{\alpha, \beta\}$ at time $t$ solve

$$
\begin{gathered}
\max _{\left(x_{i, t}, y_{i, t}\right) \in(0,+\infty)^{2}} u_{i}\left(x_{i, t}, y_{i, t}\right) \quad \text { s.t. } \\
p_{x, t} x_{i, t}+p_{y, t} y_{i, t} \leq p_{x, t} w_{x}+p_{y, t} w_{y}
\end{gathered}
$$

- the markets for the two goods clear, i.e., at time $t$ for good $j \in\{x, y\}$ it holds that

$$
a_{t} j_{\alpha, t}+\left(1-a_{t}\right) j_{\beta, t}=a_{t} w_{j}+\left(1-a_{t}\right) w_{j}=w_{j}
$$

where we denote by $j_{\alpha, t}$ and $j_{\beta, t}$ the consumption at time $t$ of good $j \in\{x, y\}$ by agents of type $\alpha$ and $\beta$, respectively.

We notice that, since utility functions are differentiably strictly increasing, problem (2) may be rewritten as

$$
\begin{aligned}
& \max _{\left(x_{i, t}, y_{i, t}\right) \in(0,+\infty)^{2}} u_{i}\left(x_{i, t}, y_{i, t}\right) \quad \text { s.t. } \\
& p_{x, t} x_{i, t}+p_{y, t} y_{i, t}=p_{x, t} w_{x}+p_{y, t} w_{y}
\end{aligned}
$$

and from the budget constraint we obtain $x_{i, t}=w_{x}+p_{t} w_{y}-p_{t} y_{i, t}$, where we set $p_{t}=p_{y, t} / p_{x, t}$. Hence, (4) simply becomes

$$
\max _{y_{i, t} \in(0,+\infty)} u_{i}\left(w_{x}+p_{t} w_{y}-p_{t} y_{i, t}, y_{i, t}\right)
$$

From here, thanks to the fact that $u_{i}$ is differentiably strictly quasiconcave, there exists a unique optimal consumption choice for good $y$, depending on $p_{t}$, that we call $y_{i, t}^{*}\left(p_{t}\right)$. Hence, the optimal consumption choice for agent $i$ of good $x$, depending on $p_{t}$, that we call $x_{i, t}^{*}\left(p_{t}\right)$, is given by $x_{i, t}^{*}\left(p_{t}\right)=$ $w_{x}+p_{t} w_{y}-p_{t} y_{i, t}^{*}\left(p_{t}\right)$. The equilibrium price $p_{t}^{*}$ can then be determined by using one of the two market clearing conditions in (3), since by Walras' law the other market clearing condition is redundant. In this manner $p_{t}^{*}$ will be influenced by the population share $a_{t}$, so that we can write $p_{t}^{*}\left(a_{t}\right)$. Inserting $p_{t}^{*}\left(a_{t}\right)$ into $x_{i, t}^{*}\left(p_{t}\right)$ and $y_{i, t}^{*}\left(p_{t}\right)$, we find the equilibrium consumption choices $x_{i, t}^{*}$ and $y_{i, t}^{*}$ for agent $i$, which will depend on $a_{t}$, as well. Indeed, using the extended approach based on first order conditions and market clearing conditions to characterize equilibria (cf. Paragraph 8.4 in Villanacci et al. 2002), it is possible to prove that, in any time period, for all $E \in \mathcal{E}$ and $a_{t} \in(0,1)$, there exists at least a market equilibrium ${ }^{6}$ (see Proposition 1) and that, for all population shares, generically in the set of economies, market equilibria are

\footnotetext{
6 We remark that the argument above suggests that market equilibria exist for any economy even when $a=0$ and $a=1$, although such extreme cases are not encompassed in Proposition 1 due to the need to deal with open sets because of the differential topology kind of proof. We also notice that considering an open interval of the form $(-\varepsilon, 1+\varepsilon)$, with $\varepsilon>0$ arbitrarily small, would not solve the issue, as some steps in the proof of Proposition 1 would not work anymore.
} 
finite and regular, i.e., they depend in a smooth manner on economies and population shares (cf. Proposition 2 where, like in Carosi et al. 2009, Gori et al. 2013, and Hoelle et al. 2015, we complement the finite-dimensional analysis performed in Villanacci et al. 2002 using the infinite-dimensional version of the Implicit Function Theorem by Gloeckner 2006). Moreover, in order to avoid indeterminacy issues, we check in Proposition 3 that a unique equilibrium exists when dealing with utility functions that yield individual demand functions with the gross substitute property. We recall that a characterization of such class of utility functions has been provided in Fisher (1972). The need to restrict our attention to utility functions which imply the existence of a unique equilibrium for every economy and for all population shares comes from the fact that, as we shall see in Subsection 2.2, in our model the attractiveness of a group depends on its social visibility level, which is obtained as a linear combination of the amount of the two goods consumed in equilibrium by the agents in that group. Hence, the existence of a unique equilibrium prevents indeterminacy issues, in which different attractiveness levels correspond to the same economy.

We stress that, although by now no dynamic aspects have been introduced in the model, and thus we are just considering a variation of the classical exchange economy setting with two consumers, in which we take into account population shares in the market clearing conditions, we need to check that all the steps in the original proofs of existence, generic regularity and uniqueness of equilibria still hold true in our framework. In particular, such verification cannot be performed on the Edgeworth box because the two groups of agents in general do not have the same numerosity. We also remark that, in order to show the existence of equilibria, we could use continuity arguments applied to the excess demand function (cf. pages 584-585 in Mas-Colell et al. 1995). However, for the homogeneity's sake with the proof of generic regularity of equilibria, we prefer to employ the extended approach to show that equilibria exist, too. The corresponding results read as follows:

Proposition 1 For every economy $E \in \mathcal{E}$, for every $t \in \mathbb{N}$ and for every population share $a_{t} \in(0,1)$, there exists at least a market equilibrium at time $t$.

Proposition 2 For every $t \in \mathbb{N}$ and for every population share $a_{t} \in(0,1)$, there exists an open and full measure ${ }^{7}$ subset $\mathcal{D}\left(a_{t}\right)$ of $\mathcal{E}$ such that, for any

\footnotetext{
7 Actually, along the proof we show the validity of a stronger result, i.e., that in any time period and for every population share, for each choice of the utility functions in $\mathcal{U}$, there exists an open and full measure subset of the space of endowments where the generic regularity property holds. In particular, the fact that the smooth dependence of equilibria holds for all share values - for all utility functions in $\mathcal{U}$ and for almost all endowment combinations - is crucial, as we will characterize market stationary equilibria in terms of population shares. Indeed, for our purposes a result more in line with the approach in Chapter 8 in Villanacci et al. (2002), where economies are identified with endowments and the utility function vector is taken as given (see in particular Paragraph 8.7 therein), would suffice. Nonetheless, the smooth dependence of equilibria on utility functions comes as a byproduct of the proof of Proposition 2, which is in line with some recent generic regularity
} 
$E \in \mathcal{D}\left(a_{t}\right)$, there is a (positive) finite number of associated market equilibria which locally smoothly depend on the elements $(E, a)$ of $\mathcal{E} \times(0,1)$.

In relation to Proposition 2, we assume that $\mathcal{E} \times(0,1)$ is endowed with the topology induced by the Hausdorff topological vector space $\mathscr{E} \times \mathbb{R}$, where $\mathscr{E}$ has been introduced in (1) and $\mathscr{E} \times \mathbb{R}$ is endowed with the product topology. In the proofs of Propositions 1 and 2 (provided in the Appendix) we will use the two following results (cf. Theorem 7.5.368 in Villanacci et al. 2002 for the former, and for the latter see Theorem 2.3 in Gloeckner 2006, of which Theorem 2 is a simplified version).

Theorem 1 Let $M, N$ be $\mathcal{C}^{2}$ boundaryless manifolds of the same dimension, $y \in N$ and $\Phi, \Gamma: M \rightarrow N$ be continuous functions. Assume that $\Gamma$ is $\mathcal{C}^{1}$ in an open neighborhood $U$ of $\Gamma^{-1}(y), y$ is a regular value for $\Gamma$ restricted to $U$, the cardinality of $\Gamma^{-1}(y)$ is finite and odd, and there exists a continuous homotopy $\Psi: M \times[0,1] \rightarrow N$ from $\Phi$ to $\Gamma$ such that $\Psi^{-1}(y)$ is compact. Then $\Phi^{-1}(y) \neq \emptyset$.

Theorem 2 Let us consider $f: O \times B \rightarrow \mathbb{R}^{n}$, where $O$ is an open subset of $\mathbb{R}^{n}$ and $B$ is an open subset of a topological Hausdorff vector space $\mathscr{B}$. Assume that $f \in \mathcal{C}^{1}\left(O \times B, \mathbb{R}^{n}\right)$ and let $\left(x_{0}, b_{0}\right) \in O \times B$ be such that $f\left(x_{0}, b_{0}\right)=0$ and $D_{x} f\left(x_{0}, b_{0}\right)$ is invertible. Then there exist $O\left(x_{0}\right) \subseteq O$ open neighborhood of $x_{0}, B\left(b_{0}\right) \subseteq B$ open neighborhood of $b_{0}$ and $\varphi: B\left(b_{0}\right) \rightarrow O\left(x_{0}\right)$ such that

1. $\varphi \in \mathcal{C}^{1}\left(B\left(b_{0}\right), O\left(x_{0}\right)\right)$,

2. $\varphi\left(b_{0}\right)=x_{0}$,

3. $\left\{(x, b) \in O\left(x_{0}\right) \times B\left(b_{0}\right): f(x, b)=0\right\}=\left\{(x, b) \in O\left(x_{0}\right) \times B\left(b_{0}\right): x=\varphi(b)\right\}$.

In regard to Theorem 2, we report some definitions related to its statement. Given a topological Hausdorff vector space $\mathscr{S}$ containing an open set $S$ and a function $g: S \rightarrow \mathbb{R}^{n}$, we say that $g \in \mathcal{C}^{0}\left(S, \mathbb{R}^{n}\right)$ if $g$ is continuous, while we say that $g \in \mathcal{C}^{1}\left(S, \mathbb{R}^{n}\right)$ if it is continuous, the limit

$$
d g(s, \sigma)=\lim _{\varepsilon \rightarrow 0} \frac{g(s+\varepsilon \sigma)-g(s)}{\varepsilon},
$$

exists for all $s \in S, \sigma \in \mathscr{S}$, and the function $d g: S \times \mathscr{S} \rightarrow \mathbb{R}^{n}$ is continuous. For further mathematical details, see Hoelle et al. (2015). In particular, as explained therein, the need to use the Gloeckner implicit function theorem comes from the fact that the $\mathcal{C}^{2}$ compact-open topology, the set of twice continuously differentiable utility functions is commonly endowed with, is not generated by a norm (see page 35 in Hirsch 1976) and thus the standard implicit function theorem cannot be applied in our framework. On the other hand, since $\mathscr{E}$ in (1) is a topological Hausdorff vector space, Theorem 2 can be used instead.

results in the general equilibrium literature (see e.g. Carosi et al. 2009 and Hoelle et al. 2013). We chose to present such version of the proposition, since we believe that a more general result about generic regularity can be of independent interest. 
In view of the introduction in Subsection 2.2 of the concepts of visibility and attractiveness of a group (cf. (6) and Definition 4), based on the equilibrium consumption choices, in order to avoid indeterminacy issues, we check in the next result that a unique equilibrium exists, even in the case of groups of agents with a different numerosity, when dealing with utility functions that yield individual demand functions with the gross substitute property. Omitting the (now irrelevant) dependence on time, we recall that, in the case of an exchange economy with two goods, the Walrasian demand function for a certain consumer of good $j \in\{x, y\}$, that we denote by $j^{*}\left(p_{x}, p_{y}\right)$, satisfies the gross substitute property if whenever $\widetilde{p}_{j}=p_{j}$ and $\widetilde{p}_{k}>p_{k}$, for $k \in\{x, y\}, k \neq j$, it holds that $j^{*}\left(\widetilde{p}_{x}, \widetilde{p}_{y}\right)>j^{*}\left(p_{x}, p_{y}\right)$.

Proposition 3 Assume that, given $E \in \mathcal{E}$, the individual demand functions, for agents $\alpha$ and $\beta$, of goods $x$ and $y$ satisfy the gross substitute property. Then, for all $E \in \mathcal{E}, t \in \mathbb{N}$ and $a_{t} \in(0,1)$, there exists a unique market equilibrium at time $t$.

\subsection{The share updating rule and the stationary equilibria}

In Subsection 2.1 we introduced the notion of market equilibrium in Definition 2 , which encompasses both the agents' choice of utility-maximizing consumption bundles and the market clearing conditions. We stress that such concept differs from the dynamic notion of equilibrium, i.e., of market stationary equilibrium. Indeed, by the latter we mean a market equilibrium in which population shares, and consequently prices and optimal consumption choices, are constant over time. In particular, shares are constant because in any period $t$ they solve the dynamic equation governing the share updating mechanism (see (8)). If shares are constant, the equilibrium price determined through the market clearing condition is constant, too, and consequently also the equilibrium consumption choices are constant.

Accordingly, we introduce the following definition of market stationary equilibrium:

Definition 3 Given the economy $E \in \mathcal{E}$, the vector $\left(a^{*}, p^{*}, x_{i}^{*}, y_{i}^{*}\right), i \in\{\alpha, \beta\}$, is a market stationary equilibrium if $a^{*} \in[0,1]$ is constant and if, given $a^{*}$, $\left(p^{*}, x_{i}^{*}, y_{i}^{*}\right), i \in\{\alpha, \beta\}$, is a market equilibrium in every time $t$.

We remark that, in order not to overburden notation and terminology, although $a^{*}$ is not part of the market equilibrium vector introduced in Definition 2 , we call the objects described in Definition 3 (market stationary) equilibria, and we use the symbol * even for the shares. We also notice that in Definition 2 there are time subscripts, missing in Definition 3, as the latter describes a stationary, time-unvarying, situation.

For the sake of brevity, when each economy admits a unique market equilibrium for all population shares, we shall identify market stationary equilibria just with the population share $a^{*}$, since it determines all other equilibrium 
components. Namely, according to what explained in Subsection 2.2, when dealing e.g. with utility functions that yield individual demand functions with the gross substitute property, it holds that, for every economy, $a^{*}$ determines a unique equilibrium price $p^{*}$, which in turns determines a unique equilibrium allocation $\left(x_{i}^{*}, y_{i}^{*}\right)_{i \in\{\alpha, \beta\}}$. In fact, in what follows we will focus on the subset $\widetilde{\mathcal{E}}$ of $\mathcal{E}$ in which utility functions yield individual demand functions with the gross substitute property, so that we will identify market stationary equilibria for the economies in $\widetilde{\mathcal{E}}$ with the corresponding population share.

The market stationary equilibria will be called trivial if they are not characterized by the coexistence between the two groups of agents, and nontrivial otherwise.

Let us now illustrate the population share evolutionary mechanism, based on a sociological payoff.

According to Heffetz (2011, 2012) some consumption activities are socially visible, where visibility is meant as the capability of the consumption of a good to convey ideas and transmit information to the other members of a social group about the status of the agents who consume that commodity. We stress that the study in Heffetz $(2011,2012)$ is motivated by the observation that many social phenomena, such as peer effects, social comparisons and fashion, are grounded on visibility. Since the various goods induce different visibility levels, we introduce the positive parameters $v_{x}$ and $v_{y}$ describing the degree of visibility that each agent derives from the consumption of a unit of commodity $x$ and of commodity $y$, respectively. Given such assumptions, we define the social visibility level $V_{i, t}$ of an agent of type $i \in\{\alpha, \beta\}$ at time $t$ as a linear combination of the units $x_{i, t}$ and $y_{i, t}$ of goods $x$ and $y$ he consumes, weighted respectively with the positive parameters $v_{x}$ and $v_{y}$, i.e., $V_{i, t}=v_{x} x_{i, t}+v_{y} y_{i, t}$. In particular, at the market equilibrium, which is unique under the maintained assumption that our utility functions yield individual demand functions with the gross substitute property, it holds that

$$
V_{i, t}^{*}=v_{x} x_{i, t}^{*}+v_{y} y_{i, t}^{*}, \text { for } i \in\{\alpha, \beta\} .
$$

For ease of notation, since we will consider visibility values just in correspondence to the market equilibrium, we will denote $V_{i, t}^{*}$ simply by $V_{i, t}$. The same remark applies to the attractiveness functions we shall introduce in Definition 4 below, which we will denote by $\mathscr{A}_{i, t}$, rather than by $\mathscr{A}_{i, t}^{*}$.

We stress that, although we assume that the parameters $v_{x}$ and $v_{y}$ cannot vanish, the case of polarized values for $v_{x}$ and $v_{y}$, in which both of them are positive but one is much larger than the other, is allowed and well approximates those frameworks in which visibility and attractiveness are produced by the consumption of a single good.

Agents' consumption choices, deriving by the underlying preference structures, give rise to different attractiveness degrees, when the preference structures of the two groups do not coincide. Indeed, in a social interaction setting, the attractiveness of a preference structure depends on its visibility in a nonmonotone manner: the social attractiveness of a preference structure is increasing in its visibility as long as the latter is not excessive, and then such 
dependence becomes decreasing.

Introducing the attractiveness $\mathscr{A}_{i, t}$ of group $i$ as a function of $V_{i, t}, i \in\{\alpha, \beta\}$, suitable hypotheses on such map are that it is bell-shaped, increasing with the visibility level up to a certain threshold value $\bar{V}$, above which it becomes decreasing in a symmetric manner with respect to $\bar{V}$, due to a congestion effect. Hence, $\mathscr{A}_{i, t}$ will be for us any differentiable and strictly decreasing function of $d\left(\bar{V}, V_{i, t}\right)=\left|\bar{V}-V_{i, t}\right|$, where $d$ denotes the Euclidean distance. In particular, in order to avoid differentiability issues, we will actually consider functions depending on $d^{2}\left(\bar{V}, V_{i, t}\right)=\left(\bar{V}-V_{i, t}\right)^{2}$. For simplicity, in what follows we will sometimes denote $d^{2}\left(\bar{V}, V_{i, t}\right)$ just by $d_{i}^{2}$. We stress that it would be possible to view the attractiveness as an index, normalizing such variable and assuming that $\mathscr{A}_{i, t}$ varies in the interval $(-1,1)$, so that negative values of the attractiveness could be interpreted as a repulsion, positive values of $\mathscr{A}_{i, t}$ would describe an attraction, and a null attractiveness would represent indifference toward a certain lifestyle. However, since such normalization would complicate the expression of attractiveness, we do not impose any restriction on the values that $\mathscr{A}_{i, t}$ may assume.

Definition 4 Given a map $f:[0,+\infty) \rightarrow(-\infty, f(0)]$, differentiable and strictly decreasing, and $\sigma>0, \bar{V}>0$, recalling the definition of $V_{i, t}$ in (6), for $i \in\{\alpha, \beta\}$, any function

$$
\mathscr{A}_{i, t}:(0,+\infty)^{3} \rightarrow\left(-\infty, \mathscr{A}_{i, t}(\bar{V})\right], \quad \mathscr{A}_{i, t}\left(V_{i, t} ; \sigma, \bar{V}\right)=f\left(\sigma d^{2}\left(\bar{V}, V_{i, t}\right)\right),
$$

will be called attractiveness of group $i$ at time $t$. The set of the admissible attractiveness functions of group $i$ at time $t$ will be denoted by $\mathcal{A}_{i, t}$.

We notice that, by definition, we have $\mathcal{A}_{\alpha, t}=\mathcal{A}_{\beta, t}$, for every $t$, and that, for $i \in\{\alpha, \beta\}$, it holds that $\mathcal{A}_{\alpha, t^{\prime}}=\mathcal{A}_{\alpha, t^{\prime \prime}}$, for all $t^{\prime}, t^{\prime \prime} \in \mathbb{N}$. Hence, we will denote the set of the admissible attractiveness functions simply by $\mathcal{A}$. In particular, in order to simplify computations and, most importantly, in view of making the share updating rule depending just on a comparison between the distance of the visibility levels for the two groups from the threshold value $\bar{V}$, we will assume that the same map $f$ in (7) describes the attractiveness of both groups $\alpha$ and $\beta$. Nonetheless, we will specify after the main results we obtain what it would be possible to infer when allowing for different attractiveness formulations between groups.

As concerns the parameters, we remark that, by (7), the attractiveness depends in an explicit manner only on $\sigma$. The dependence of $\mathscr{A}_{i, t}$ on the other model parameters is indirect, and occurs due to the presence of $V_{i, t}$. The parameter $\sigma$ in (7) describes the sensitivity of the attractiveness $\mathscr{A}_{i, t}$, for $i \in\{\alpha, \beta\}$, with respect to the distance between the visibility level $V_{i, t}$ and the threshold level $\bar{V}$. In particular, a visibility level coinciding with $\bar{V}$ allows maximizing the attractiveness degree. We stress that when $\sigma \rightarrow 0$ agents are insensitive even to a large distance between $V_{i, t}$ and $\bar{V}$, and $\mathscr{A}_{i, t}$ tends towards its maximum value $\mathscr{A}_{i, t}(\bar{V})$. When instead $\sigma \rightarrow+\infty$, even a small, but still positive, distance between $V_{i, t}$ and $\bar{V}$ leads to a very low attractiveness level because of an excessive sensitivity to such distance. 
The attractiveness functions satisfying the assumptions in Definition 4 are suitable to describe, in relation to group $i \in\{\alpha, \beta\}$, both the bandwagon behavior, which occurs as long as $V_{i, t}<\bar{V}$, and the snob behavior, which occurs when $V_{i, t}>\bar{V}$. Indeed, according to Simmel (1904), two contrasting tendencies operate in determining people behavior towards fashion. On the one hand, to follow fashion makes people feel accepted and socially integrated, answering to their innate tendency for conformity. This generates imitation of others, i.e., the so called bandwagon behavior. Such phenomenon is reproduced by $\mathscr{A}_{i, t}$ as long as $V_{i, t}$ is smaller than $\bar{V}$, when increasing values for $V_{i, t}$ imply higher and higher attractiveness degrees $\mathscr{A}_{i, t}$. On the other hand, when the visibility of a group becomes excessive, a congestion effect arises and the people wish for distinction predominates. That phenomenon is known as snob behavior and it is reproduced by $\mathscr{A}_{i, t}$ when $V_{i, t}$ is larger than $\bar{V}$, because in this regime an increase in the visibility level $V_{i, t}$ leads to a decrease in $\mathscr{A}_{i, t}$. Namely, according to Vigneron and Johnson (1999), on the basis of the empirical literature, too, in the context of luxury and prestige-seeking consumption agents may oscillate between snob and bandwagon behaviors. Those two opposite forces, imitation and distinction, drive the fashion cycle, which for us emerges at the aggregate level as a continuous oscillation of the attractiveness and of the shares of the two groups, while on the individual level it is characterized by oscillatory consumption choices, presenting booms and busts, over the two goods for the agents belonging to the two groups. We shall find evidence of such phenomenon in Section 3 (cf. Figures 1 and 3 therein), when considering Stone-Geary utility functions and for the formulation of the attractiveness map already dealt with in Naimzada and Pireddu (2018). ${ }^{8}$

The share of agents which choose to belong to a given group in the next period depends on the present attractiveness levels of the two groups. More precisely, following Branch and McGough (2008), Cabrales and Sobel (1992), Hofbauer and Weibull (1996), Nachbar (1990), Sandholm (2010), Taylor and Jonker (1978), we do consider the discrete exponential replicator mechanism to formalize the population share updating rule, so that the evolution of the fraction $a_{t}$ of agents of type $\alpha$ is described by the discrete choice model

$$
\begin{aligned}
a_{t+1} & =\frac{a_{t} \exp \left(\mu \mathscr{A}_{\alpha, t}\right)}{a_{t} \exp \left(\mu \mathscr{A}_{\alpha, t}\right)+\left(1-a_{t}\right) \exp \left(\mu \mathscr{A}_{\beta, t}\right)} \\
& =\frac{a_{t}}{a_{t}+\left(1-a_{t}\right) \exp \left(\mu\left(\mathscr{A}_{\beta, t}-\mathscr{A}_{\alpha, t}\right)\right)},
\end{aligned}
$$

where $\mu$ is a positive parameter measuring the sensitivity of the share formation mechanism to the difference in the groups attractiveness levels. When we will need to underline the dependence, for $i \in\{\alpha, \beta\}$, of $\mathscr{A}_{i, t}$ and of $V_{i, t}$ on $a_{t}$, we will also write $\mathscr{A}_{i, t}\left(a_{t}\right)$ and $V_{i, t}\left(a_{t}\right)$, respectively, or, at the market stationary equilibria, simply $\mathscr{A}_{i}(a)$ and $V_{i}(a)$ (see for instance the map $g$

\footnotetext{
8 A more detailed bifurcation analysis, performed for several parameter configurations and for the formulations of the attractiveness maps introduced in Naimzada and Pireddu (2019a), too, can be found in the Appendix of Naimzada and Pireddu (2019b).
} 
in (10), whose fixed points are the system market stationary equilibria). ${ }^{9}$ In particular, recalling the definition of visibility in (6) and that, by Proposition 2 and Footnote 7 , the equilibrium consumption choices depend smoothly on $a_{t} \in(0,1)$, we have that also $V_{i, t}, i \in\{\alpha, \beta\}$, depend smoothly on $a_{t} \in(0,1)$, for every $t$, and that, in correspondence to a market stationary equilibrium, $V_{i}(a), i \in\{\alpha, \beta\}$, depend in a smooth way on $a \in(0,1)$.

By (7) and (8), we obtain

$$
a_{t+1}=g\left(a_{t}\right)
$$

where the one-dimensional map $g:[0,1] \rightarrow \mathbb{R}$ is defined as

$$
g(a)=\frac{a}{a+(1-a) \exp \left(\mu\left(f\left(\sigma d^{2}\left(\bar{V}, V_{\beta}(a)\right)\right)-f\left(\sigma d^{2}\left(\bar{V}, V_{\alpha}(a)\right)\right)\right)\right)} .
$$

We stress that if $\mu \rightarrow 0$, independently of $V_{\alpha}$ and $V_{\beta}$, then $a_{t+1}=a_{t}$, for all $t$, and thus there is no evolution, the population shares remain unchanged with respect to the initial ones, as agents are insensitive to the attractiveness degrees of the two groups; when instead $\mu \rightarrow+\infty$, agents are extremely sensitive to the social attractiveness of the groups and they instantaneously move towards the "best" one, i.e., the one which gave a visibility level closer to $\bar{V}$.

Let us start our analysis by deriving the expressions of the market stationary equilibria for $(9)$.

Proposition 4 Given the economy $E \in \widetilde{\mathcal{E}}$ and the attractiveness $\mathscr{A}_{i, t}\left(V_{i, t} ; \sigma, \bar{V}\right)$ $=f\left(\sigma d^{2}\left(\bar{V}, V_{i, t}\right)\right) \in \mathcal{A}$, for $i \in\{\alpha, \beta\}$, equation (9) admits as market stationary equilibria, in addition to the trivial $a=0, a=1$, also all solutions in $(0,1)$ to the equation $V_{\alpha}(a)=V_{\beta}(a)$, if any, as well as all solutions in $(0,1)$ to the equation $\left(V_{\alpha}(a)+V_{\beta}(a)\right) / 2=\bar{V}$, if any.

We remark that the condition $\left(V_{\alpha}+V_{\beta}\right) / 2=\bar{V}$ in Proposition 4 means that $\bar{V}$ is the midpoint between $V_{\alpha}$ and $V_{\beta}$. Hence, at the nontrivial market stationary equilibria, the population share of group $\alpha$ has to make $V_{\alpha}$ and $V_{\beta}$ coincide or $V_{\alpha}$ and $V_{\beta}$ have to lie at the same distance from $\bar{V}$, but on its opposite sides. We also notice that assuming in Proposition 4 that $\mathscr{A}_{\alpha}=f_{\alpha}\left(\sigma d^{2}\left(\bar{V}, V_{\alpha}\right)\right)$ and $\mathscr{A}_{\beta}=f_{\beta}\left(\sigma d^{2}\left(\bar{V}, V_{\beta}\right)\right)$, for some $f_{\alpha} \neq f_{\beta}$, with $f_{\alpha}, f_{\beta}$ differentiable and strictly decreasing maps, we then find that, in addition to the trivial $a=0, a=1$, the market stationary equilibria are the solutions in $(0,1)$ to the equation $f_{\alpha}\left(\sigma d^{2}\left(\bar{V}, V_{\alpha}\right)\right)=f_{\beta}\left(\sigma d^{2}\left(\bar{V}, V_{\beta}\right)\right)$, if any. We finally stress that if both $\mathscr{A}_{\alpha}$ and $\mathscr{A}_{\beta}$ were described by the same map $f$, but if $\mathscr{A}_{\alpha}=f\left(\sigma_{\alpha} d^{2}\left(\bar{V}, V_{\alpha}\right)\right)$ and $\mathscr{A}_{\beta}=f\left(\sigma_{\beta} d^{2}\left(\bar{V}, V_{\beta}\right)\right)$, for some $\sigma_{\alpha} \neq \sigma_{\beta}$, then the nontrivial market stationary equilibria would be given by all solutions in $(0,1)$ to the equation $d\left(\bar{V}, V_{\alpha}\right)=$ $d\left(\bar{V}, V_{\beta}\right) \sqrt{\frac{\sigma_{\beta}}{\sigma_{\alpha}}}$, i.e., to the equations $V_{\alpha}-\sqrt{\frac{\sigma_{\beta}}{\sigma_{\alpha}}} V_{\beta}=\bar{V}\left(1-\sqrt{\frac{\sigma_{\beta}}{\sigma_{\alpha}}}\right)$ and $V_{\alpha}+$ $\sqrt{\frac{\sigma_{\beta}}{\sigma_{\alpha}}} V_{\beta}=\bar{V}\left(1+\sqrt{\frac{\sigma_{\beta}}{\sigma_{\alpha}}}\right)$. Namely, setting $\sigma_{\alpha}=\sigma_{\beta}$ in such expressions, we find again the results obtained in Proposition 4.

\footnotetext{
9 We remark that $V_{i}$, and consequently $\mathscr{A}_{i}$, also depend on the economy $\widetilde{\mathcal{E}}$, which together with $a$ determines the market equilibrium. However, in order not to overburden notation, we will not make such dependence explicit.
} 
In the next result we analytically investigate the local stability of the market stationary equilibria for the map $g$ in (10).

Proposition 5 The equilibrium $a=0$ is locally asymptotically stable for the map $g$ in $(10)$ if $\left(V_{\beta}(0)-\bar{V}\right)^{2}<\left(V_{\alpha}(0)-\bar{V}\right)^{2}$.

The equilibrium $a=1$ is locally asymptotically stable for map $g$ if $\left(V_{\alpha}(1)-\right.$ $\bar{V})^{2}<\left(V_{\beta}(1)-\bar{V}\right)^{2}$.

When $\mathscr{A}_{i}, i \in\{\alpha, \beta\}$, do not depend on $\mu$, calling $\hat{a}$ any solution to the equation $V_{\alpha}(a)=V_{\beta}(a)$, if it exists in $(0,1)$, the equilibrium $a=\hat{a}$ is locally asymptotically stable for map $g$ if $\left(V_{\beta}(\hat{a})-\bar{V}\right)\left(V_{\beta}^{\prime}(\hat{a})-V_{\alpha}^{\prime}(\hat{a})\right)<0$ and

$$
\mu<\hat{\mu}=\frac{1}{\left.\sigma \hat{a}(1-\hat{a}) \frac{\partial \mathscr{A}_{\beta}(a)}{\partial\left(\sigma d_{\beta}^{2}\right)}\right|_{a=\hat{a}}\left(V_{\beta}(\hat{a})-\bar{V}\right)\left(V_{\beta}^{\prime}(\hat{a})-V_{\alpha}^{\prime}(\hat{a})\right)} .
$$

In particular, a flip bifurcation occurs at $a=\hat{a}$ if $\mu=\hat{\mu}$.

If $\left(V_{\beta}(\hat{a})-\bar{V}\right)\left(V_{\beta}^{\prime}(\hat{a})-V_{\alpha}^{\prime}(\hat{a})\right)>0$, then $\hat{a} \in(0,1)$ is unstable.

When $\mathscr{A}_{i}, i \in\{\alpha, \beta\}$, do not depend on $\mu$, calling $\tilde{a}$ any solution to the equation $\left(V_{\alpha}(a)+V_{\beta}(a)\right) / 2=\bar{V}$, if it exists in $(0,1)$, the equilibrium $a=\tilde{a}$ is locally asymptotically stable for map $g$ if $\left(\bar{V}-V_{\alpha}(\tilde{a})\right)\left(V_{\alpha}^{\prime}(\tilde{a})+V_{\beta}^{\prime}(\tilde{a})\right)<0$ and

$$
\mu<\tilde{\mu}=\frac{1}{\left.\sigma \tilde{a}(1-\tilde{a}) \frac{\partial \mathscr{A}_{\beta}(a)}{\partial\left(\sigma d_{\beta}^{2}\right)}\right|_{a=\tilde{a}}\left(\bar{V}-V_{\alpha}(\tilde{a})\right)\left(V_{\alpha}^{\prime}(\tilde{a})+V_{\beta}^{\prime}(\tilde{a})\right)} .
$$

In particular, a flip bifurcation occurs at $a=\tilde{a}$ if $\mu=\tilde{\mu}$.

If $\left(\bar{V}-V_{\alpha}(\tilde{a})\right)\left(V_{\alpha}^{\prime}(\tilde{a})+V_{\beta}^{\prime}(\tilde{a})\right)>0$, then $\tilde{a} \in(0,1)$ is unstable.

We notice that if we assumed $\mathscr{A}_{\alpha}=f_{\alpha}\left(\sigma d^{2}\left(\bar{V}, V_{\alpha}\right)\right)$ and $\mathscr{A}_{\beta}=f_{\beta}\left(\sigma d^{2}\left(\bar{V}, V_{\beta}\right)\right)$, for some $f_{\alpha} \neq f_{\beta}$, with $f_{\alpha}, f_{\beta}$ differentiable and strictly decreasing maps, we would find that $a=0$ is locally asymptotically stable for the map $g$ in (10) if $\mathscr{A}_{\beta}(0)>\mathscr{A}_{\alpha}(0)$ and that $a=1$ is locally asymptotically stable if $\mathscr{A}_{\beta}(1)<\mathscr{A}_{\alpha}(1)$. However, in such more general setting, those conditions on attractiveness could not be translated into conditions on visibility and thus they would remain more vague. Moreover, if $f_{\alpha}, f_{\beta}$ do not depend on $\mu$, calling $\bar{a}$ any solution to the equation $f_{\alpha}\left(\sigma d^{2}\left(\bar{V}, V_{\alpha}\right)\right)=f_{\beta}\left(\sigma d^{2}\left(\bar{V}, V_{\beta}\right)\right)$, if it exists in $(0,1)$, we find that $a=\bar{a}$ is locally asymptotically stable for the map $g$ in (10) if $\left.\frac{\partial \mathscr{A}_{\beta}(a)}{\partial\left(\sigma d_{\beta}^{2}\right)}\right|_{a=\bar{a}}\left(V_{\beta}(\bar{a})-\bar{V}\right) V_{\beta}^{\prime}(\bar{a})-\left.\frac{\partial \mathscr{A}_{\alpha}(a)}{\partial\left(\sigma d_{\alpha}^{2}\right)}\right|_{a=\bar{a}}\left(V_{\alpha}(\bar{a})-\bar{V}\right) V_{\alpha}^{\prime}(\bar{a})>0$ and

$$
\mu<\bar{\mu}=\frac{1}{\sigma \bar{a}(1-\bar{a})\left(\left.\frac{\partial \mathscr{A}_{\beta}(a)}{\partial\left(\sigma d_{\beta}^{2}\right)}\right|_{a=\bar{a}}\left(V_{\beta}(\bar{a})-\bar{V}\right) V_{\beta}^{\prime}(\bar{a})-\left.\frac{\partial \mathscr{A}_{\alpha}(a)}{\partial\left(\sigma d_{\alpha}^{2}\right)}\right|_{a=\bar{a}}\left(V_{\alpha}(\bar{a})-\bar{V}\right) V_{\alpha}^{\prime}(\bar{a})\right)} .
$$

In particular, a flip bifurcation occurs at $a=\bar{a}$ if $\mu=\bar{\mu}$.

If $\left.\frac{\partial \mathscr{A}_{\beta}(a)}{\partial\left(\sigma d_{\beta}^{2}\right)}\right|_{a=\bar{a}}\left(V_{\beta}(\bar{a})-\bar{V}\right) V_{\beta}^{\prime}(\bar{a})-\left.\frac{\partial \mathscr{A}_{\alpha}(a)}{\partial\left(\sigma d_{\alpha}^{2}\right)}\right|_{a=\bar{a}}\left(V_{\alpha}(\bar{a})-\bar{V}\right) V_{\alpha}^{\prime}(\bar{a})<0$, then $a=$ $\bar{a}$ is unstable for all positive values of $\mu$.

We also stress that it is not possible to derive general conditions similar to (11) and (12) when investigating the local stability of the nontrivial equilibria with respect to $\sigma$. Namely, even if $V_{i}, i \in\{\alpha, \beta\}$, do not depend on $\sigma$, such parameter 
is usually present in the expression of $\left.\frac{\partial \mathscr{A}_{\beta}(a)}{\partial\left(\sigma d_{\beta}^{2}\right)}\right|_{a=\hat{a}}$ and of $\left.\frac{\partial \mathscr{A}_{\beta}(a)}{\partial\left(\sigma d_{\beta}^{2}\right)}\right|_{a=\tilde{a}}$, as it is easy to check, for instance, with the formulations for attractiveness in (16) and in (17). In fact, with the Gaussian formulation in (17), for suitable parameter configurations, there may not exist threshold stability values analogous to those in (11) and (12) with respect to $\sigma$, since e.g. $a=\hat{a} \in(0,1)$ may be stable for any $\sigma>0 .{ }^{10}$

In agreement with Proposition 5 , in Section 3 we will consider $\mu$ as bifurcation parameter, so that the theoretical investigation performed so far will serve as a guideline for the qualitative bifurcation analysis we shall conduct below.

\section{Bifurcation analysis and illustration of the fashion cycle in the case of Stone-Geary utility functions}

In the present section we perform a qualitative bifurcation analysis, investigating the stability gain/loss of stationary equilibria and the emergence/disappearance of periodic and chaotic attractors on varying the sensitivity parameter $\mu$. Indeed, assuming a constitutive heterogeneity between groups in terms of the structure of preferences, our aim is that of investigating the model asymptotic heterogeneity, i.e., we discuss the possible dynamics arising when choosing initial conditions characterized by the coexistence between heterogeneous agents in view of understanding whether the initial heterogeneity eventually disappears and, in case it persists, if it is stationary or oscillatory in nature, of periodic or chaotic kind. In order to make the heterogeneity between groups explicit in our setting, we need to specify an analytical formulation for the utility functions.

Recalling that in Naimzada and Pireddu (2018, 2019a) agents' preferences were described by Cobb-Douglas utility functions, we will now deal with StoneGeary utility functions ${ }^{11}$, which generalize the previously considered maps, still yielding, for suitable parameter values, to individual demand functions with the gross substitute property. ${ }^{12}$ In this manner, since Stone-Geary utility functions satisfy Assumptions $(A 1)-(A 4)$ in Definition 1, according to Proposition 3 for all endowments and population shares there exists a unique equilibrium as described in (14) and thus no indeterminacy issues on visibility and attractiveness arise.

The formulation of the Stone-Geary utility functions over the two consumption

\footnotetext{
10 This happens for instance with the parameter configuration considered in Section 3, when fixing $c_{i}=d_{i}=0$, for $i \in\{\alpha, \beta\}, \mu=2$ and letting $\sigma>0$ free to vary. See Scenario A in Naimzada and Pireddu (2019b) for the corresponding details.

11 The Stone-Geary utility functions were derived by Geary (1950) in a comment on an earlier work, while Stone (1954) estimated the Linear Expenditure System, arising from the utility functions in (13).

12 Although such feature is mentioned in Fisher (1972) for Stone-Geary utility functions when the coefficients $c_{i}, d_{i}$ are non-negative, for $i \in\{\alpha, \beta\}$, a direct proof using the expression for the individual demand functions in (14) shows that the gross substitute property holds also in the case of negative coefficients, as long as their value is not excessively large in absolute value. See the discussion after (14) for more details.
} 
goods $x$ and $y$ is given by

$$
U_{i}(x, y)=\left(x+c_{i}\right)^{i}\left(y+d_{i}\right)^{1-i}, \text { for } i \in\{\alpha, \beta\} \text {, with } 0<\beta<\alpha<1 .
$$

Such maps are certainly well defined when $c_{i} \geq 0$ and $d_{i} \geq 0$, case considered in Fisher (1972), and indeed for $c_{i}=d_{i}=0$ we obtain the Cobb-Douglas utility functions. However, as we shall see below, in order to ensure that the equilibrium consumption levels in (14) are positive, $c_{i}$ and $d_{i}$ can not assume excessively large positive values. As concerns the negativity of $c_{i}$ and $d_{i}$, albeit not affecting the positivity of the equilibrium consumption levels in (14), it may prevent the individual demand functions from having the gross substitute property. Both features, i.e., the positivity of the equilibrium consumption levels and individual demand functions with the gross substitute property, are ensured for values of $c_{i}$ and $d_{i}$ not too large in absolute value (cf. (15)). We stress that allowing for negative values of $c_{i}$ and $d_{i}$ is important from an interpretative viewpoint. Namely, Stone-Geary functions are often used to model problems involving subsistence levels of consumption. In these cases, a certain minimal level of some good has to be consumed, irrespective of its price or of the consumer's income.

The next analysis is performed in terms of the relative price $p_{t}=p_{y, t} / p_{x, t}$, where $p_{x, t}>0$ and $p_{y, t}>0$ are the prices at time $t$ for goods $x$ and $y$, respectively. Solving the consumer maximization problem in (4) with the StoneGeary utility functions and using one market clearing condition in (3), as optimal equilibrium price we find

$$
p_{t}^{*}=\frac{a_{t}(1-\alpha)\left(w_{x}+c_{\alpha}\right)+\left(1-a_{t}\right)(1-\beta)\left(w_{x}+c_{\beta}\right)}{a_{t}\left(\alpha w_{y}+d_{\alpha}\right)+\left(1-a_{t}\right)\left(\beta w_{y}+d_{\beta}\right)}
$$

and the consumption equilibrium quantities of the two goods for agents of type $\alpha$ or $\beta$ are given by

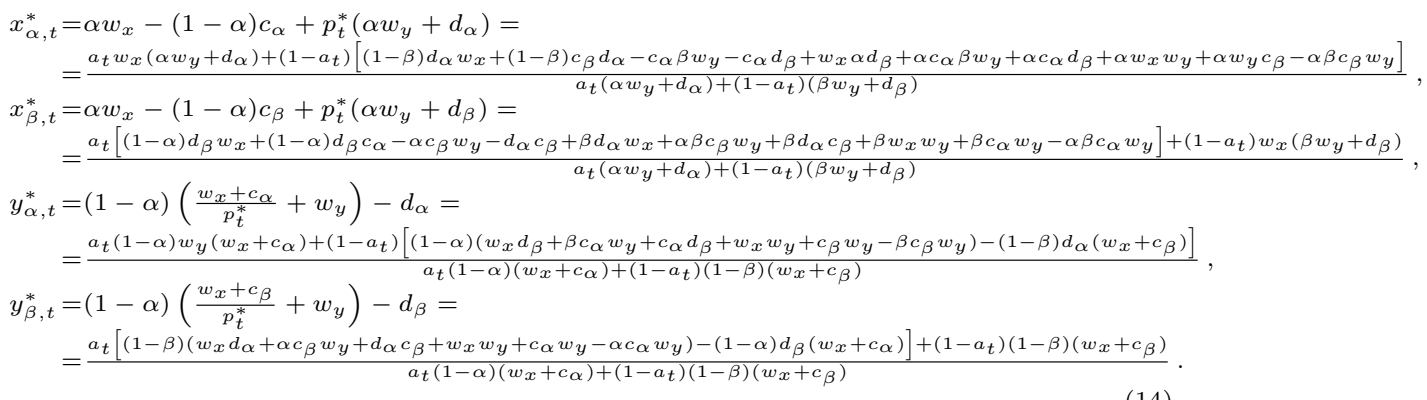

Recalling (6), in such context the social visibility level $V_{i, t}$ of an agent of type $i \in\{\alpha, \beta\}$ at time $t$ is given by $V_{i, t}=v_{x} x_{i, t}^{*}+v_{y} y_{i, t}^{*}$, with the expressions for $x_{i, t}^{*}$ and $y_{i, t}^{*}$ just derived and for values of the visibility weight parameters $v_{x}$ and $v_{y}$ to be fixed below.

From (14) we immediately find that a sufficient condition for the positivity of $x_{i, t}^{*}, i \in\{\alpha, \beta\}$, is $c_{i}<\frac{\alpha w_{x}}{1-\alpha}$, while a sufficient condition for the positivity of $y_{i, t}^{*}, i \in\{\alpha, \beta\}$, is $d_{i}<(1-\alpha) w_{y}$. On the other hand, recalling that $p_{t}=$ 
$p_{y, t} / p_{x, t}$, from (14) we obtain $\alpha w_{y}+d_{i}>0$ as sufficient condition for $x_{i, t}^{*}, i \in$ $\{\alpha, \beta\}$, to display the gross substitute property, and $w_{x}+c_{i}>0$ as sufficient condition for $y_{i, t}^{*}, i \in\{\alpha, \beta\}$, to display the gross substitute property. Hence, the positivity of the equilibrium consumption levels and individual demand functions with the gross substitute property are simultaneously guaranteed for

$$
-w_{x}<c_{i}<\frac{\alpha w_{x}}{1-\alpha}, \quad-\alpha w_{y}<d_{i}<(1-\alpha) w_{y}, \quad i \in\{\alpha, \beta\} .
$$

As regards the attractiveness function, we will deal with the bell-shaped formulation considered in Naimzada and Pireddu (2018), i.e.,

$$
\mathscr{A}_{i, t}^{\prime}\left(V_{i, t} ; \sigma, \bar{V}\right)=\frac{1}{1+\sigma\left(\bar{V}-V_{i, t}\right)^{2}},
$$

while we refer the interested reader to Naimzada and Pireddu (2019b), where we also work with the formulations for the attractiveness functions considered in Naimzada and Pireddu (2019a), i.e., the Gaussian map

$$
\mathscr{A}_{i, t}^{\prime \prime}\left(V_{i, t} ; \sigma, \bar{V}\right)=\exp \left(-\sigma\left(\bar{V}-V_{i, t}\right)^{2}\right) \text {, }
$$

and the parabolic function

$$
\mathscr{A}_{i, t}^{\prime \prime \prime}\left(V_{i, t} ; \sigma, \bar{V}\right)=1-\sigma\left(\bar{V}-V_{i, t}\right)^{2} .
$$

The choice of dealing, here or in Naimzada and Pireddu (2019b), with the above attractiveness maps comes from a simple observation. In Naimzada and Pireddu (2018, 2019a) we considered Cobb-Douglas utility functions and the bifurcation analysis was performed in terms of the heterogeneity degree between groups of agents $\Delta=\alpha-\beta$. Since the Stone-Geary utility functions are a generalization of the Cobb-Douglas utility functions, when considering in (13) $c_{i}=d_{i}=0, i \in\{\alpha, \beta\}$, and using the sensitivity measure $\mu$ as bifurcation parameter, we can complement the investigation performed in Naimzada and Pireddu $(2018,2019 \mathrm{a})$ as a byproduct of the bifurcation analysis we are going to sketch below, and which is fully developed in Naimzada and Pireddu (2019b) for the case $c_{i}=d_{i}=0, i \in\{\alpha, \beta\}$.

We stress that the attractiveness functions in (16)-(18) satisfy the conditions in Definition 4. Namely, they are differentiable, bell-shaped, increasing with the visibility level $V_{i, t}, i \in\{\alpha, \beta\}$, up to the threshold value $\bar{V}$, after which they become decreasing in $V_{i, t}$ in a symmetric manner with respect to $\bar{V}$, due to their dependence on $d^{2}\left(\bar{V}, V_{i, t}\right)$. We recall that $\sigma$ is a positive parameter describing the sensitivity of the attractiveness with respect to the distance between the visibility level $V_{i, t}$ and the threshold visibility level $\bar{V}$, while $\mu$, that we shall employ as bifurcation parameter, measures the sensitivity of the share formation mechanism to the difference in the preference structures attractiveness levels (see also the discussion after (10)).

In view of the qualitative bifurcation analysis that we will briefly outline below, referring to Naimzada and Pireddu (2019b) for further details, we explain which are the stationary equilibria of our system and we illustrate some of 
their features.

As concerns the trivial equilibria, according to Proposition $5, a=0$ is locally asymptotically stable for the map $g$ in $(10)$ if $\left(V_{\beta}(0)-\bar{V}\right)^{2}<\left(V_{\alpha}(0)-\bar{V}\right)^{2}$ and $a=1$ is locally asymptotically stable for map $g$ if $\left(V_{\alpha}(1)-\bar{V}\right)^{2}<\left(V_{\beta}(1)-\bar{V}\right)^{2}$. Hence, the stability of the trivial equilibria is influenced neither by the value of $\mu$, nor by the formulation of the map describing attractiveness. In particular, $a=0$ and $a=1$ are locally asymptotically stable for every positive value of $\mu$, both for the configuration considered below and in the frameworks analyzed in Naimzada and Pireddu (2019b).

In regard to the nontrivial stationary equilibria of (9), according to Proposition 4 , they are given by the solutions belonging to the interval $(0,1)$ to $V_{\alpha}(a)=V_{\beta}(a)$, as well as by the solutions in $(0,1)$ to the equation $\left(V_{\alpha}(a)+\right.$ $\left.V_{\beta}(a)\right) / 2=\bar{V}$. Hence, their number and expression are independent of the chosen formulation for attractiveness and of the value of $\mu$. On the other hand, recalling (11) and (12), the stability of the nontrivial stationary equilibria is influenced by the value of $\mu$ and by the formulation of the map describing attractiveness. Indeed, the latter affects the stability threshold value of nontrivial equilibria when considering $\mu$ as bifurcation parameter. Moreover, in analogy with the findings in Naimzada and Pireddu (2018, 2019a), we expect that varying $\mu$ the global dynamics may differ according to the formulation for attractiveness, e.g. due to the occurrence of subcritical or supercritical flip bifurcations at the nontrivial equilibria. We find a confirmation of such conjecture in Naimzada and Pireddu (2019b).

The parameter configuration we will deal with is given by $v_{x}=0.9, w_{x}=$ $0.2, v_{y}=0.15, w_{y}=2, \beta=0.1, \alpha=0.9, \sigma=8, \bar{V}=0.8$, while we let $\mu$ free to vary. ${ }^{13}$ We stress that such parameter values coincide with those considered in Naimzada and Pireddu (2018, 2019a), even if, as explained above, in those works we used $\Delta=\alpha-\beta$ as bifurcation parameter, while setting $\mu=6.5$. As concerns the new parameters $c_{i}$ and $d_{i}$ characterizing the Stone-Geary utility functions, from (15) we obtain the following bounds: ${ }^{14}$

$$
-0.2<c_{i}<1.8, \quad-1.8<d_{i}<0.2, \quad i \in\{\alpha, \beta\} .
$$

Hence, in regard to the values of $c_{i}$ and $d_{i}$ many different choices are possible. The simplest one is characterized by $c_{i}=d_{i}=0$, for $i \in\{\alpha, \beta\}$, so that the Stone-Geary utility functions reduce to Cobb-Douglas utility functions. Such scenario may be treated as a benchmark context, which allows for a comparison between the bifurcation analysis performed in Naimzada and Pireddu (2018, 2019a) in terms of $\Delta=\alpha-\beta$ and here in terms of $\mu$. A second possible choice

\footnotetext{
13 We here consider polarized values for $v_{x}$ and $v_{y}$, as both of them are positive but one is much larger than the other. As observed in Section 2, such case approximates those frameworks in which visibility and attractiveness are produced by the consumption of a single good. However, our results hold true also for more balanced values of $v_{x}$ and $v_{y}$.

14 We remark that the symmetry between the bounds of $c_{i}$ and $d_{i}$ is caused by the fact that, for the parameter configuration we deal with, it holds that $w_{x}=(1-\alpha) w_{y}$. Of course, such peculiarity does not affect the outcomes. Indeed, in the scenario that we will consider below the parameters $c_{i}$ and $d_{i}$ will not bear any symmetry.
} 


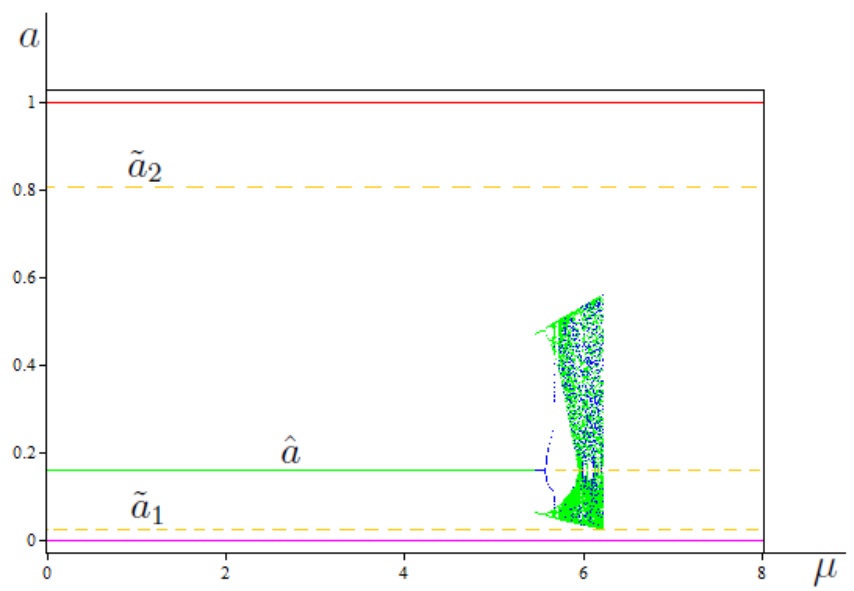

Fig. 1 The bifurcation diagram of $g$ for $c_{\alpha}=-0.19, c_{\beta}=-0.14, d_{\alpha}=-0.3, d_{\beta}=$ $-0.16, \mu \in(0,8)$ and the attractiveness $\mathscr{A}^{\prime}$ in $(16)$. We denote in magenta $a=0$, in red $a=1$, in blue (green) the points generated by the initial condition $a_{0}=0.3437\left(a_{0}=0.8\right)$ and in orange $a=\hat{a}$ when it is no more stable, as well as the unstable equilibria $a=\tilde{a}_{1}$ and $a=\tilde{a}_{2}$. Solid (dashed) lines refer to stable (unstable) equilibria and cycles

is characterized by positive values for all parameters in the Stone-Geary utility functions, while a third possible framework is characterized by negative values for all parameters in the Stone-Geary utility functions. For the brevity's sake, we here discuss just the third framework, which is both the best grounded from an interpretative viewpoint, as well as the most interesting in terms of the arising dynamics. In particular, we will consider $c_{\alpha}=-0.19, c_{\beta}=-0.14, d_{\alpha}=$ $-0.3, d_{\beta}=-0.16$, introducing only those aspects needed to illustrate the emergence of the fashion cycle in our model for the attractiveness formulation in (16). The analysis of the first two scenarios, as well as the remaining details regarding the third scenario, in correspondence to each of the attractiveness formulations in (16)-(18) can be found in Naimzada and Pireddu (2019b). ${ }^{15}$ In Figure 1 we report the bifurcation diagram of $g$ for $\mu \in(0,8)$. In addition to the trivial equilibria, which are locally asymptotically stable for all positive values of $\mu$, we notice that for small values of $\mu$ just $a=\hat{a}$ is stable. When $\mu$ increases, an external attractor, born via a fold bifurcation of the second iterate of the map $g$ as a period-two cycle and then undergoing a sequence of period-doubling bifurcations leading to chaos, coexists first with $a=\hat{a}$ and next with the period-two cycle following the supercritical flip bifurcation occurring at $a=\hat{a}$ for $\mu=\hat{\mu}=5.549$. For still larger values of $\mu$, the latter period-two cycle disappears through a reverse fold bifurcation of the second iterate of $g$. Moreover, the external attractor, which for increasing values of parameter $\mu$ from being a two-piece chaotic attractor becomes a one-piece chaotic attractor, disappears for $\mu=6.228$ due to a contact bifurcation with

15 In regard to the second scenario, in Naimzada and Pireddu (2019b) we deal with $c_{\alpha}=$ $0.3, c_{\beta}=0.12, d_{\alpha}=0.1, d_{\beta}=0.15$. 


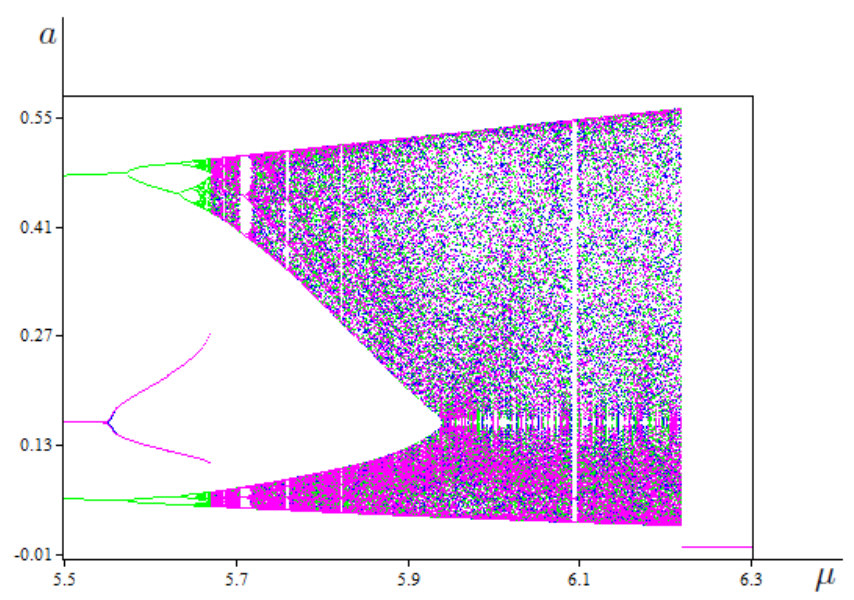

Fig. 2 An enlargement of the bifurcation diagram in Figure 1 for $\mu \in(5.5,6.3)$, where we denote in blue the points generated by the initial condition $a_{0}=0.159$, in magenta the points generated by the initial condition $a_{0}=0.161$ and in green the points generated by the initial condition $a_{0}=0.8$.

$a=\tilde{a}_{1}$. See also Figure 2, where we provide an enlargement, for $\mu \in(5.5,6.3)$, of the bifurcation diagram in Figure 1, in which we do not report the unstable equilibria and the trivial ones. After the contact bifurcation occurring between the chaotic attractor and $a=\tilde{a}_{1}$, the only attractors are given by $a=0$ and $a=1$. We stress that, due to the disappearance of the internal period-two cycle, suddenly oscillations in the agents' consumption choices and in the population shares become strong. We recall that this kind of framework is a mixture between the outcomes found in Naimzada and Pireddu (2018, 2019a). Namely, like in Figure 5 in Naimzada and Pireddu (2019a), in Figure 1 above the nontrivial equilibrium $a=\hat{a}$ undergoes a supercritical flip bifurcation. However, the period-two cycle following it, rather than undergoing a classical cascade of flip bifurcations to chaos, disappears, and this suddenly leads to wide oscillations, similar to those which follow the subcritical flip bifurcation in Figure 5 in Naimzada and Pireddu (2018) and in Figure 1 in Naimzada and Pireddu (2019a). As in such settings, we here find interesting multistability phenomena, involving equilibria, as well as periodic or chaotic attractors, suitable to represent the variety of historical experiences across different countries in relation to the approach they adopt towards consumption choices and fashion.

Summarizing, Figure 1 highlights that in our model fashion cycle dynamics may be generated by intermediate values of the sensitivity parameter $\mu$. Indeed, when $\mu$ is too small, the only attractors are steady states and this excludes the possibility of any sort of non-convergent dynamics. When $\mu$ increases, the nontrivial equilibrium $a=\hat{a}$ loses stability and the period-two cycle following it coexists with an external attractor which, contrarily to the internal period-two cycle, persists, giving rise to periodic and chaotic dynam- 
ics. However, when the sensitivity measure becomes excessive, no oscillatory behaviors occur anymore, as the main chaotic attractor disappears due to a contact bifurcation with one of the nontrivial equilibria, and thus the system, according to the chosen initial condition, asymptotically converges toward one or the other of the trivial steady states, characterized by the presence of a unique group of agents. In fact, even for lower values of the sensitivity parameter $\mu$, trajectories will tend toward one of the three or four coexisting attractors according to the chosen initial datum. In particular, when the initial conditions, which represent a summary of the past history, are excessively close to the extreme values $a=0$ and $a=1$, trajectories are attracted by one of the two trivial equilibria, and a preference structure totally prevails over the other. However, when the initial datum is not too close to $a=0$ and $a=1$, for intermediate values of $\mu$, our dynamical system may lead to outcomes characterized by the coexistence among heterogeneous agents. Due to the combined effect of the price formation mechanism, of the social interaction mechanism, according to which consumption choices produce visibility, and of the evolutionary mechanism, based on the relative attractiveness of the different lifestyles, the group coexistence may be stationary or oscillatory in nature, both periodic, in a neighborhood of the flip bifurcation, and erratic, due to the presence of the chaotic attractor. When the group coexistence is oscillatory, it displays the recurrent behavior typical of the fashion cycle, characterized by booms and busts. We report in Figure 3, for the same parameter configuration considered in Figure 1 with $\mu=6.1$ and for the periods $t \in[200,300]$, the time series for all the relevant variables. Namely, the fashion cycle for us emerges at the aggregate level as a continual oscillation of the attractiveness and of the shares of the two groups, while on the individual level it is characterized by oscillatory consumption choices over the two goods for the agents of the two groups.

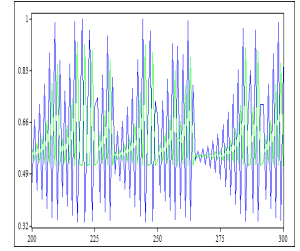

(a)

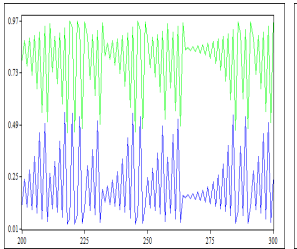

(b)

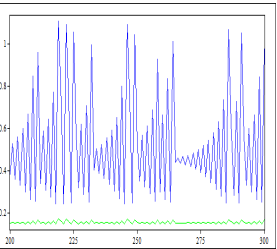

(c)

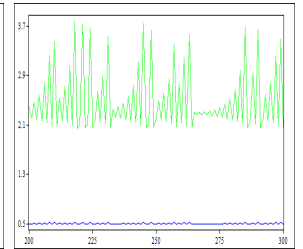

(d)

Fig. 3 The time series corresponding to the periods $t \in[200,300]$ for $\mathscr{A}_{\alpha, t}^{\prime}$ (in blue) and $\mathscr{A}_{\beta, t}^{\prime}$ (in green) in (a), for $a_{t}$ (in blue) and $b_{t}=1-a_{t}$ (in green) in (b), for $x_{\alpha, t}^{*}$ (in blue) and $x_{\beta, t}^{*}$ (in green) in (c), for $y_{\alpha, t}^{*}$ (in blue) and $y_{\beta, t}^{*}$ (in green) in (d), for the same parameter configuration considered in Figure 1 , with $\mu=6.1$ and $a_{0}=0.4$

We stress that, although the proof of chaos performed in Naimzada and Pireddu (2018) using the method of the turbulent maps in Block and Coppel (1992), working with homoclinic orbits, can be directly transposed to the 
present setting, the social and economic interpretation of the main scenarios provided in Naimzada and Pireddu (2018, 2019a) cannot be precisely repeated here. For such reason, we conclude the present section by briefly reporting the interpretation of the most important phenomena we found, in view of better understanding the functioning of the model dynamics. The main scenarios in Figure 1 are: the convergence towards a trivial steady state, so that the agents' original heterogeneity contained in the initial condition does not persist; the convergence towards a nontrivial steady state, that is a static form of heterogeneity; the convergence towards a period-two cycle, which represents the simplest case of oscillatory behavior, that encompasses agents' heterogeneity and in which population shares, prices and consumption bundles vary over time. We notice that the other possible scenarios may be explained combining the arguments that we shall employ below.

As concerns the convergence towards a trivial steady state, let us consider for instance $\mu=2$ and $a_{0}=0.85$ and let us clarify why the system converges towards $a=1$. Since $V_{\alpha}<\bar{V}<V_{\beta}$, with $\left(V_{\alpha}-\bar{V}\right)^{2}<\left(V_{\beta}-\bar{V}\right)^{2}$, we expect an increase in the share of the agents of group $\alpha$ and, consequently, a raise in the aggregate demand of commodity $x$, agents of group $\alpha$ have a stronger preference for. Indeed, we do observe a decrease in the relative price $p$. The optimal consumption quantities for the two goods are determined and, since the value of $x_{\alpha}^{*}$ decreases more than $x_{\beta}^{*}$ and $v_{x}$ is much larger than $v_{y}$, the value of $V_{\alpha}$ raises less than the value of $V_{\beta}$, so that the ordering among $V_{\alpha}, \bar{V}$ and $V_{\beta}$ is maintained but the distance $\left(V_{\beta}-\bar{V}\right)^{2}$ increases, while the distance $\left(V_{\alpha}-\bar{V}\right)^{2}$ decreases. The repetition of such process eventually leads to the extinction of the agents of group $\beta$. The explanation of the scenario which leads to the convergence towards $a=0$ is omitted, as it is completely symmetric.

In regard to the convergence towards a nontrivial steady state, let us consider for instance $\mu=2$ and $a_{0}=0.7$ and let us clarify why the system tends to $a=\hat{a}=0.160$. Since $V_{\alpha}<\bar{V}<V_{\beta}$, with $\left(V_{\alpha}-\bar{V}\right)^{2}>\left(V_{\beta}-\bar{V}\right)^{2}$, we expect a decrease in the share of the agents of group $\alpha$ and, consequently, a fall in the aggregate demand of commodity $x$. Indeed, we do observe a raise in the relative price $p$. The optimal consumption quantities for the two goods are determined and, since the value of $x_{\alpha}^{*}$ increases more than $x_{\beta}^{*}$ and $v_{x}$ is much larger than $v_{y}$, the value of $V_{\alpha}$ increases while the value of $V_{\beta}$ decreases, so that the new ordering among $V_{\alpha}, \bar{V}$ and $V_{\beta}$ is given by $V_{\alpha}<V_{\beta}<\bar{V}$. Since $V_{\beta}$ is closer than $V_{\alpha}$ to $\bar{V}$, the share of agents of type $\alpha$ falls. The repetition of such process leads to visibility values which satisfy $V_{\alpha}<V_{\beta}<\bar{V}$, with $V_{\alpha}$ that increases and $V_{\beta}$ that decreases, so that the share of agents of type $\alpha$ is progressively reduced, as long as it holds that $V_{\alpha}=V_{\beta}=0.48$ in correspondence to the nontrivial steady state $a=\hat{a}=0.160$.

Let us finally provide the explanation of a framework which leads to the convergence towards a period-two cycle. Considering e.g. $\mu=5.5$ and starting from $a_{0}=0.06$, we have that the agents of type $\alpha$ are few and thus we expect that the aggregate demand for commodity $x$ is low. Indeed, we do observe a high value for the relative price $p$. The optimal consumption quantities for the 
two goods are determined and, since $p$ is high, the value of $x_{\alpha}^{*}$ is high, too. Due to the fact that $v_{x}$ is much larger than $v_{y}$, we find that $V_{\beta}<V_{\alpha}<\widehat{V}$. Since the distance between $V_{\alpha}$ and $\widehat{V}$ is smaller than the distance between $V_{\beta}$ and $\widehat{V}$, the share of agents of type $\alpha$ raises and exceeds $a=0.47$. We then expect that the aggregate demand for commodity $x$ is higher than before. Indeed, we observe a lower value for the relative price $p$. The optimal consumption quantities for the two goods are determined and, since $p$ is lower now, the value of $x_{\alpha}^{*}$ decreases. As $v_{x}$ is still much larger than $v_{y}$, we find that $V_{\alpha}<V_{\beta}<\widehat{V}$. Since $V_{\beta}$ is closer to $\widehat{V}$ than $V_{\alpha}$, the share of agents of type $\alpha$ decreases and is again near $a=0.06$, giving rise to the period-two cycle, whose values are $a=0.062$ and $a=0.476$.

\section{Conclusions}

In the present work, in order to represent the fashion cycle, we proposed a discrete-time exchange economy evolutionary model with two groups of agents, in which the reproduction level of a group is related to its attractiveness degree, that depends on the social visibility level, determined by the consumption choices of the agents in that group. In particular, in agreement with the empirical literature (see Vigneron and Johnson 1999) we assumed that agents exhibit both bandwagon and snob attitudes. Namely, according to Simmel (1904), imitation and distinction drive the fashion cycle. Like in Naimzada and Pireddu (2019a), we encompassed such behavioral assumption in our model dealing with generic bell-shaped attractiveness functions for the two groups of agents, that are increasing for low visibility levels and decreasing when the visibility of the group exceeds a given threshold value, due to a congestion effect. However, differently from that paper, rather than considering just Cobb-Douglas utility functions, we let utility functions free to vary in a suitable set of maps. This increased generality in the class of considered utility functions urged us to investigate not only the dynamic features of our setting, but also to study the existence and the generic regularity of the market equilibria, which link the equilibrium price and the optimal consumption choices to population shares. In order to illustrate the general results we obtained on equilibria, as well as to investigate the model dynamics, we added a simulative section where we considered Stone-Geary utility functions, which generalize the Cobb-Douglas utility functions, and where we opted for the formulation of the attractiveness map dealt with in Naimzada and Pireddu (2018). Like in that setting, also in the present framework we observed interesting multistability phenomena, involving equilibria, as well as periodic or chaotic attractors. In particular, thanks to the combined action of the price formation mechanism, of the share updating rule and of the agents' social interaction, with the alternation between the bandwagon and snob behaviors, the dynamic coexistence between groups and the oscillatory nature of the agents' consumption activities display the recurrent dynamic behavior typical of the fashion cycle, characterized by booms and busts. 
As concerns future study directions, our setting could be employed to represent the fashion cycle in frameworks with capital accumulation, such as the OLG model by Diamond (1965).

A further extension of our setting would consist in assuming two different time scales for consumption choices and for the evolutionary mechanism. Indeed, since the consumption activities require less time than the updating process of the population shares, it would be suitable to deal with a continuous-time formulation for the consumption choices and a discrete-time formulation for the share updating rule. This would lead to the study of a hybrid dynamical system, like those analyzed e.g. in Cavalli and Naimzada (2016) and in Cavalli et al. (2018).

Alternatively, we could suppose that the utility of some consumers depends also on the attractiveness level of their own group, in order to investigate whether the more "rational" agents, which are aware of the functioning of the share updating mechanism, perform better from an evolutionary viewpoint. Finally, modifying the argument of agents' utility functions so as to enter a setting with strategic interaction, like those considered in Heifetz et al. (2007a, 2007b), and displaying strategic complementarity and/or substitutability, we could investigate the possible dynamical effects produced by two groups endowed with heterogeneous structures of preferences in those contexts, too.

Funding: This research did not receive any specific grant.

Conflict of Interest: The authors declare that they have no conflict of interest.

\section{Acknowledgments}

The authors thank the anonymous Referees for the helpful and valuable comments.

\section{References}

Antoci, A., Brugnano, L., Galeotti, M.: Sustainability, indeterminacy and oscillations in a growth model with environmental assets. Nonlinear Anal. Real World Appl. 5, 571-587 (2004)

Benhabib, J., Day, R.H.: Rational choice and erratic behaviour. Rev. Econ. Stud. 48, 459-471 (1981)

Bischi, G.-I., Radi, D.: An extension of the Antoci-Dei-Galeotti evolutionary model for environment protection through financial instruments. Nonlinear Anal. Real World Appl. 13, 432-440 (2012)

Block, L.S., Coppel, W.A.: Dynamics in One Dimension. Springer Lecture Notes, 1513, Springer Verlag, Berlin (1992)

Branch, W.A., McGough, B.: Replicator dynamics in a Cobweb model with rationally heterogeneous expectations. J. Econ. Behav. Org. 65, 224-244 (2008) 
Cabrales, A., Sobel, J.: On the limit points of discrete selection dynamics. J. Econ. Theory 57, 407-419 (1992)

Carosi, L., Gori, M., Villanacci, A.: Endogenous restricted participation in general financial equilibrium. J. Math. Econ. 45, 787-806 (2009)

Caulkins, J.P., Hartl, R.F., Kort, P.M., Feichtinger, G.: Explaining fashion cycles: Imitators chasing innovators in product space. J. Econ. Dyn. Control 31, 1535-1556 (2007)

Cavalli, F., Naimzada, A.: A multiscale time model with piecewise constant argument for a boundedly rational monopolist. J. Difference Equ. Appl. 22, 1480-1489 (2016)

Cavalli, F., Naimzada, A., Sodini, M.: Oligopoly models with different learning and production time scales. Decis. Econ. Finan. 41, 297-312 (2018)

Chang, J., Stauber, R.: Evolution of preferences in an exchange economy. Econ. Lett. 103, 131-134 (2009)

Coelho, P.R.P., McClure, J.E.: Toward an economic theory of fashion. Econ. Inq. 31, 595-608 (1993)

Corneo, G., Jeanne, O.: Segmented communication and fashionable behavior. J. Econ. Behav. Organ. 39, 371-385 (1999)

Day, R.H.: Rational choice and economic behavior. Theor. Decis. 1, 229-251 (1970)

Di Giovinazzo, V., Naimzada, A.: A model of fashion: Endogenous preferences in social interaction. Econ. Model. 47, 12-17 (2015)

Diamond, P.A.: National debt in a neoclassical growth model. Am. Econ. Rev. 55, 1126-1150 (1965)

Ekaterinchuk, E., Jungeilges, J., Ryazanova, T., Sushko, I.: Dynamics of a minimal consumer network with uni-directional influence. J. Evol. Econ. $27,831-857$ (2017)

Ekaterinchuk, E., Jungeilges, J., Ryazanova, T., Sushko, I.: Dynamics of a minimal consumer network with bi-directional influence. Commun. Nonlinear Sci. Numer. Simul. 58, 107-118 (2018)

Fisher, F.M.: Gross substitutes and the utility function. J. Econ. Theory 4, 82-87 (1972)

Frijters, P.: A model of fashions and status. Econ. Model. 15, 501-517 (1998)

Gaertner, W.: A dynamic model of interdependent consumer behavior. Zeitschr. f. Nationalökonomie 34, 327-344 (1974)

Gaertner, W.: Periodic and aperiodic consumer behavior. Appl. Math. Comput. 22, 233-254 (1987)

Gaertner, W., Jungeilges, J.: A non-linear model of interdependent consumer behaviour. Econ. Lett. 27, 145-150 (1988)

Gaertner, W., Jungeilges, J.: "Spindles" and coexisting attractors in a dynamic model of interdependent consumer behavior: A note. J. Econ. Behav. Organ. 21, 223-231 (1993)

Gaertner, W., Jungeilges, J.: A model of interdependent consumer behavior: Nonlinear dynamics in $\mathbb{R}^{2}$. In: Eichhorn, W. (ed.) Models and Measurement of Welfare and Inequality, pp. 941-962. Springer-Verlag, Berlin (1994) 
Gardini, L., Sushko, I., Matsuyama, K.: 2D discontinuous piecewise linear map: Emergence of fashion cycles. Chaos 28, 055917 (2018). https://doi.org/10.1063/1.5018588

Geary, R.C.: A note on "A constant-utility index of the cost of living". Rev. Econ. Studies 18, 65-66 (1950)

Gloeckner, H.: Implicit functions from topological vector spaces to Banach spaces. Isr. J. Math. 155, 205-252 (2006)

Gori, M., Pireddu, M., Villanacci, A.: Regularity and Pareto improving on financial equilibria with price-dependent borrowing restrictions. Res. Econ. 67, 100-110 (2013)

Gori, M., Pireddu, M., Villanacci, A.: Existence of financial equilibria with endogenous short selling restrictions and real assets. Decisions in Economics and Finance 37, 349-371 (2014)

Heffetz, O.: A test of conspicuous consumption: visibility and income elasticities. Rev. Econ. Stat. 93, 1101-1117 (2011)

Heffetz, O.: Who sees what? Demographics and the visibility of consumer expenditures. J. Econ. Psychol. 33, 801-818 (2012)

Heifetz, A., Shannon, C., Spiegel, Y.: The dynamic evolution of preferences. Econ. Theory 32, 251-286 (2007a)

Heifetz, A., Shannon, C., Spiegel, Y.: What to maximize if you must. J. Econ. Theory 133, 31-57 (2007b)

Hirsch, M.: Differential Topology. Springer, New York (1976)

Hoelle, M., Pireddu, M., Villanacci, A.: Incomplete financial markets with real assets and endogenous credit limits. J. Econ. 117 (2015). https://doi.org/10.1007/s00712-015-0438-4

Hofbauer, J., Weibull, J.: Evolutionary selection against dominated strategies. J. Econ. Theory 71, 558-573 (1996)

Karni, E., Schmeidler, D.: Fixed preferences and changing tastes. Am. Econ. Rev. 80, 262-267 (1990)

Leibenstein, H.: Bandwagon, snob and Veblen effects in the theory of consumers. Q. J. Econ. 65, 183-207 (1950)

Lloyd, N.G.: Degree Theory, Cambridge Tracts in Mathematics, No. 73. Cambridge University Press, Cambridge (1978)

Mas-Colell, A., Whinston, M.D., Green, J.R.: Microeconomic Theory. Oxford University Press, New York (1995)

Matsumoto, A.: Let it be: chaotic price instability can be beneficial. Chaos Solitons Fractals 18, 745-758 (2003)

Matsuyama, K.: Custom versus fashion: Path-dependence and limit cycles in a random matching game. Working Paper in Economics, E-92-11, Hoover Institution; Northwestern University CMS-EMS DP \# 1030 (1991)

Nachbar, J.H.: "Evolutionary" selection dynamics in games: Convergence and limit properties. Int. J. Game Theory 19, 59-89 (1990)

Naimzada, A., Pireddu, M.: Endogenous evolution of heterogeneous consumers preferences: Multistability and coexistence between groups. Econ. Lett. 142, 22-26 (2016) 
Naimzada, A., Pireddu, M.: Fashion cycle dynamics in a model with endogenous discrete evolution of heterogeneous preferences. Chaos 28, 055907 (2018). https://doi.org/10.1063/1.5024931

Naimzada, A., Pireddu, M.: Fashion cycle dynamics induced by agents' heterogeneity for generic bell-shaped attractiveness functions. J. Differ. Equ. Appl. (2019a). https://doi.org/10.1080/10236198.2019.1627344

Naimzada, A., Pireddu, M.: A general equilibrium evolutionary model with generic utility functions and generic bell-shaped attractiveness maps, generating fashion cycle dynamics. Extended version available as: University of Milan Bicocca Department of Economics, Management and Statistics Working Paper No. 401 (2019b)

Naimzada, A., Pireddu, M.: The first fundamental theorem of welfare in a general equilibrium evolutionary setting. University of Milan Bicocca Department of Economics, Management and Statistics Working Paper No. 415 (2019c)

Naimzada, A., Sacco, P., Sodini, M.: Wealth-sensitive positional competition as a source of dynamic complexity in OLG models. Nonlinear Anal. Real World Appl. 14, 1-13 (2013)

Pesendorfer, W.: Design innovation and fashion cycles. Am. Econ. Rev. 85, 771-792 (1995)

Sandholm, W.H.: Population Games and Evolutionary Dynamics. MIT Press, Cambridge (2010)

Sharkovsky, A.N., Kolyada, S.F., Sivak, A.G., Fedorenko, V.V.: Dynamics of One-Dimensional Maps. Kluwer Academic, Boston (1997)

Simmel, G.: Fashion. Int. Q. 10, 130-155 (1904)

Stone, R.: Linear expenditure systems and demand analysis: An application to the pattern of British demand. Econ. J. 64, 511-527 (1954)

Taylor, P.D., Jonker, L.B.: Evolutionarily stable strategies and game dynamics. Math. Biosci. 40, 145-156 (1978)

Veblen, T.: The Theory of the Leisure Class: an Economic Study of Institutions. Macmillan, New York (1899)

Vigneron, F., Johnson, L.: A review and a conceptual framework of prestigeseeking consumer behavior. Acad. Mark. Sci. Rev. 3 (1999)

Villanacci, A., Carosi, L., Benevieri, P., Battinelli, A.: Differential Topology and General Equilibrium with Complete and Incomplete Markets. Springer US (2002)

Wiggins, S.: Introduction to Applied Nonlinear Dynamical Systems and Chaos, Texts in Applied Mathematics, second ed. Springer, New York (2003)

Zhang, W.-B.: Fashion with snobs and bandwagoners in a three-type households and three-sector neoclassical growth model. Revista Mexicana de Economía y Finanzas 11, 1-19 (2016)

Zhang, W.-B.: Fashion and business cycles with snobs and bandwagoners in a multi-sector growth model. J. Bus. 2, 1-13 (2017) 


\section{A Proof of the analytical results}

Proof of Proposition 1: Since the arguments we shall employ are independent of the considered time period, in order not to overburden notation, we will omit the subscript $t$, as well as the stars, which will refer to the Pareto Optimal allocation ${ }^{16}$ only.

We define $\theta=\left(p_{x}, p_{y},\left(x_{i}, y_{i}\right)_{i \in\{\alpha, \beta\}}\right) \in \Theta=(0,+\infty)^{6}$ and, according to Definition 2 , we say that $\theta \in \Theta$ is a market equilibrium given $E \in \mathcal{E}$ and $a \in(0,1)$ if, for every $i \in\{\alpha, \beta\}$, $\left(x_{i}, y_{i}\right)$ solves problem $(4)$ at $\left(p_{x}, p_{y}, E\right)$ and $\left(x_{i}, y_{i}\right)_{i \in\{\alpha, \beta\}}$ satisfies market clearing conditions $(3)$ at $(E, a)$.

We denote by $\Theta(E, a)$ the set of market equilibria for $E$ and $a$, and we introduce

$$
\Theta_{\mathrm{n}}(E, a)=\left\{\theta \in \Theta(E, a): p_{x}=1\right\}
$$

that is, the set of normalized ${ }^{17}$ market equilibria for $E$ and $a$. We will show that $\Theta_{\mathrm{n}}(E, a) \neq$ $\emptyset$, for every $(E, a) \in \mathcal{E} \times(0,1)$.

As one Walras' law holds true in our model, just one market clearing condition in Definition 2 is significant. To fix ideas, we will focus on the equation for commodity $y$, i.e., on

$$
a y_{\alpha}+(1-a) y_{\beta}=w_{y}
$$

The extended system for our economy with two groups of agents, whose numerosity may not coincide, reads as

$$
\left\{\begin{array}{l}
\frac{\partial u_{\alpha}}{\partial x_{\alpha}}\left(x_{\alpha}, y_{\alpha}\right)-\lambda_{\alpha} p_{x}=0 \\
\frac{\partial u_{\alpha}}{\partial y_{\alpha}}\left(x_{\alpha}, y_{\alpha}\right)-\lambda_{\alpha} p_{y}=0 \\
-p_{x} x_{\alpha}-p_{y} y_{\alpha}+p_{x} w_{x}+p_{y} w_{y}=0 \\
\frac{\partial u_{\beta}}{\partial x_{\beta}}\left(x_{\beta}, y_{\beta}\right)-\lambda_{\beta} p_{x}=0 \\
\frac{\partial u_{\beta}}{\partial y_{\beta}}\left(x_{\beta}, y_{\beta}\right)-\lambda_{\beta} p_{y}=0 \\
-p_{x} x_{\beta}-p_{y} y_{\beta}+p_{x} w_{x}+p_{y} w_{y}=0 \\
a y_{\alpha}+(1-a) y_{\beta}-w_{y}=0 \\
p_{x}-1=0
\end{array}\right.
$$

Since we are going to study market equilibria in terms of first-order conditions associated with households' maximization problems and (significant) market clearing conditions, we define

$$
\xi=\left(p_{x}, p_{y},\left(x_{i}, y_{i}, \lambda_{i}\right)_{i \in\{\alpha, \beta\}}\right) \in(0,+\infty)^{8},
$$

and the function

$$
\mathcal{F}:(0,+\infty)^{8} \times \mathcal{E} \times(0,1) \rightarrow \mathbb{R}^{8}, \quad \mathcal{F}(\xi, E, a)=\text { lhs of }(19) .
$$

Given $(E, a) \in \mathcal{E} \times(0,1)$, it is immediate to prove that if $\theta=\left(p_{x}, p_{y},\left(x_{i}, y_{i}\right)_{i \in\{\alpha, \beta\}}\right)$ belongs to $\Theta_{\mathrm{n}}(E, a)$, then there exists the vector $\left(\lambda_{\alpha}, \lambda_{\beta}\right) \in(0,+\infty)^{2}$ such that $\xi=$ $\left(p_{x}, p_{y},\left(x_{i}, y_{i}, \lambda_{i}\right)_{i \in\{\alpha, \beta\}}\right) \in(0,+\infty)^{8}$ solves the system $\mathcal{F}(\xi, E, a)=0$. Vice versa, if $\xi=$ $\left(p_{x}, p_{y},\left(x_{i}, y_{i}, \lambda_{i}\right)_{i \in\{\alpha, \beta\}}\right) \in(0,+\infty)^{8}$ solves $\mathcal{F}(\xi, E, a)=0$, then $\left(p_{x}, p_{y},\left(x_{i}, y_{i}\right)_{i \in\{\alpha, \beta\}}\right) \in$ $\Theta_{\mathrm{n}}(E)$.

16 See Naimzada and Pireddu (2019c) for the corresponding definition and for the proof, in our framework, of the first fundamental theorem of welfare economics, according to which every (stationary) equilibrium allocation is Pareto Optimal.

Since the employed concept of Pareto Optimality concerns one single period at a time, we will use the expression "instantaneous Pareto Optimal allocation" in the present work.

17 We stress that, differently from what done in Villanacci et al. (2002), we normalize the price of the first commodity, rather than of the last one. Of course, this change does not affect the validity of the results. 
Let us then show that for all $(E, a) \in \mathcal{E} \times(0,1)$ there exists $\xi \in(0,+\infty)^{8}$ which solves system $\mathcal{F}(\xi, E, a)=0$. Fixing $(E, a) \in \mathcal{E} \times(0,1)$, we define

$$
F:(0,+\infty)^{8} \rightarrow \mathbb{R}^{8}, \quad F(\xi)=\mathcal{F}(\xi, E, a) .
$$

Let us also introduce the homotopy

$$
H:(0,+\infty)^{8} \times[0,1] \rightarrow \mathbb{R}^{8}, \quad H(\xi, \tau)=\text { lhs of }(21),
$$

with

$$
\left\{\begin{array}{l}
\frac{\partial u_{\alpha}}{\partial x_{\alpha}}\left(x_{\alpha}, y_{\alpha}\right)-\lambda_{\alpha} p_{x}=0 \\
\frac{\partial u_{\alpha}}{\partial y_{\alpha}}\left(x_{\alpha}, y_{\alpha}\right)-\lambda_{\alpha} p_{y}=0 \\
-p_{x} x_{\alpha}-p_{y} y_{\alpha}+p_{x}\left((1-\tau) w_{x}+\tau x_{\alpha}^{*}\right)+p_{y}\left((1-\tau) w_{y}+\tau y_{\alpha}^{*}\right)=0 \\
\frac{\partial u_{\beta}}{\partial x_{\beta}}\left(x_{\beta}, y_{\beta}\right)-\lambda_{\beta} p_{x}=0 \\
\frac{\partial u_{\beta}}{\partial y_{\beta}}\left(x_{\beta}, y_{\beta}\right)-\lambda_{\beta} p_{y}=0 \\
-p_{x} x_{\beta}-p_{y} y_{\beta}+p_{x}\left((1-\tau) w_{x}+\tau x_{\beta}^{*}\right)+p_{y}\left((1-\tau) w_{y}+\tau y_{\beta}^{*}\right)=0 \\
a y_{\alpha}+(1-a) y_{\beta}-\left((1-\tau) w_{y}+\tau\left(a y_{\alpha}^{*}+(1-a) y_{\beta}^{*}\right)\right)=0 \\
p_{x}-1=0,
\end{array}\right.
$$

where $\left(x_{i}^{*}, y_{i}^{*}\right)_{i \in\{\alpha, \beta\}} \in(0,+\infty)^{4}$ is an instantaneous Pareto Optimal allocation, whose existence can be proven as in Section 8.5 in Villanacci et al. (2002). In particular, denoting by $r$ the vector of the total resources associated with $E$, we have $r=\left(w_{x}, w_{y}\right) \in(0,+\infty)^{2}$ and, denoting by $\underline{U}^{r}$ the set of utility level vectors attainable with resources $r$ in correspondence to $E \in \mathcal{E}$ and $a \in(0,1)$, we have

$\underline{U}^{r}=\left\{\left(\underline{u}_{\alpha}, \underline{u}_{\beta}\right) \in \mathbb{R}^{2}: \exists\left(x_{\alpha}, x_{\beta}, y_{\alpha}, y_{\beta}\right) \in(0,+\infty)^{4}\right.$ s.t. $\left.\begin{array}{l}a j_{\alpha}+(1-a) j_{\beta}=w_{j}, \text { for } j \in\{x, y\}, \\ \text { and } u_{i}\left(x_{i}, y_{i}\right)-\underline{u}_{i}=0, \text { for } i \in\{\alpha, \beta\}\end{array}\right\}$.

We notice that $H(\xi, 0)=F(\xi), \forall \xi \in(0,+\infty)^{8}$. Setting

$$
G:(0,+\infty)^{8} \rightarrow \mathbb{R}^{8}, \quad G(\xi)=H(\xi, 1),
$$

it holds that $F, H$ and $G$ are continuous functions. If we prove that, for some $\widehat{\xi} \in(0,+\infty)^{8}$,

$$
\begin{aligned}
& G^{-1}(0)=\{\widehat{\xi}\} \text { and } G \text { is } \mathcal{C}^{1} \text { in an open neighborhood of } \widehat{\xi}, \\
& D_{\xi} G(\widehat{\xi}) \text { is not singular, } \\
& H^{-1}(0) \text { is compact, }
\end{aligned}
$$

then Theorem 1 can be applied with the identifications $M=(0,+\infty)^{8}, N=\mathbb{R}^{8}, y=0 \in$ $\mathbb{R}^{8}, \Phi=F, \Gamma=G, \Psi=H$, to get $F^{-1}(0) \neq \emptyset$, so that a market equilibrium exists in correspondence to the fixed $(E, a) \in \mathcal{E} \times(0,1)$.

The proof of conditions (22), (23) and (24) follows by standard arguments and it is omitted.

Proof of Proposition 2: Since the arguments we shall employ are independent of the considered time period, like in the proof of Proposition 1, in order not to overburden notation, we will omit the subscript $t$.

Recalling the definition of the map $\mathcal{F}$ in (20), it is evident that $\mathcal{F}$ is continuous. In order to apply Theorem 2 with the identifications $f=\mathcal{F}, \mathbb{R}^{n}=\mathbb{R}^{8}, O=(0,+\infty)^{8}, B=\mathcal{E} \times(0,1), \mathscr{B}=$ $\mathscr{E} \times \mathbb{R}$, with $\mathscr{E}$ as in (1), we have to check that $\mathcal{F} \in \mathcal{C}^{1}\left((0,+\infty)^{8} \times \mathcal{E} \times(0,1), \mathbb{R}^{8}\right)$ according to the definition in (5), i.e., that

$$
d \mathcal{F}:\left((0,+\infty)^{8} \times \mathcal{E} \times(0,1)\right) \times\left(\mathbb{R}^{8} \times \mathscr{E} \times \mathbb{R}\right) \rightarrow \mathbb{R}^{8}
$$

is well defined and continuous. Considering any $(\xi, E, a) \in(0,+\infty)^{8} \times \mathcal{E} \times(0,1)$ and $(\nu, \eta, \kappa) \in$ $\mathbb{R}^{8} \times \mathscr{E} \times \mathbb{R}$, it holds indeed that the limit

$$
d \mathcal{F}(\xi, E, a)=\lim _{\varepsilon \rightarrow 0} \frac{\mathcal{F}(\xi+\varepsilon \nu, E+\varepsilon \eta, a+\varepsilon \kappa)-\mathcal{F}(\xi, E, a)}{\varepsilon}
$$


exists, and it is easy to check that the map $d \mathcal{F}$ is continuous.

Next, we want to show that for every $a \in(0,1)$ the set

$$
\mathcal{D}(a)=\left\{E \in \mathcal{E}: \mathcal{F}(\xi, E, a)=0 \Rightarrow \operatorname{det} D_{\xi} \mathcal{F}(\xi, E, a) \neq 0\right\}
$$

is an open and full measure subset of $\mathcal{E}$. Namely, applying Theorem 2, we obtain the smooth dependence of the market equilibria associated to all population shares $a \in(0,1)$ and to any economy $E \in \mathcal{D}(a)$ on the elements $(E, a) \in \mathcal{E} \times(0,1)$.

Let us then sketch the proof that, for every $a \in(0,1), \mathcal{D}(a)$ is an open and full measure subset of $\mathcal{E}$.

The openness of $\mathcal{D}(a)$ follows by the closedness of the complement set $\mathcal{E} \backslash \mathcal{D}(a)$, due to the continuity of the involved functions. In order to show that $\mathcal{D}(a)$ is a full measure subset of $\mathcal{E}$, it suffices to show that for every $\left(u_{\alpha}, u_{\beta}, a\right) \in \mathcal{U}^{2} \times(0,1)$ the set

$\mathcal{D}\left(u_{\alpha}, u_{\beta}, a\right)=\left\{\left(w_{x}, w_{y}\right) \in(0,+\infty)^{2}: \mathcal{F}\left(\xi, u_{\alpha}, u_{\beta}, w_{x}, w_{y}, a\right)=0 \Rightarrow \operatorname{det} D_{\xi} \mathcal{F}(\xi, E, a) \neq 0\right\}$ is a full measure subset of $(0,+\infty)^{2}$. Since it can be proven that 0 is a regular value for the map

$$
\widetilde{\mathcal{F}}:(0,+\infty)^{8} \times(0,+\infty)^{2} \rightarrow \mathbb{R}^{8}, \quad \widetilde{\mathcal{F}}\left(\xi, w_{x}, w_{y}\right)=\mathcal{F}\left(\xi, u_{\alpha}, u_{\beta}, w_{x}, w_{y}, a\right),
$$

then, by a transversality result (cf. Theorem 6.3.294 in Villanacci et al. 2002), there exists a full measure subset $\widetilde{\mathcal{D}}\left(u_{\alpha}, u_{\beta}, a\right)$ of $(0,+\infty)^{2}$ such that, for all $\left(w_{x}, w_{y}\right) \in \widetilde{\mathcal{D}}\left(u_{\alpha}, u_{\beta}, a\right), 0$ is a regular value for the map $\widetilde{\mathcal{F}}\left(\cdot, w_{x}, w_{y}\right)$. As $\widetilde{\mathcal{D}}\left(u_{\alpha}, u_{\beta}, a\right) \subseteq \mathcal{D}\left(u_{\alpha}, u_{\beta}, a\right)$, it follows that $\mathcal{D}\left(u_{\alpha}, u_{\beta}, a\right)$ is a full measure subset of $(0,+\infty)^{2}$. Consequently, for every $a \in(0,1), \mathcal{D}(a)$ is a full measure subset of $\mathcal{E}$.

The finiteness of the number of market equilibria associated to an element $(a, E)$, with $a \in(0,1)$ and $E \in \mathcal{D}(a)$, comes now from the fact that, as it is easy to check, the projection

$$
\pi: \mathcal{F}^{-1}(0) \rightarrow \mathcal{E} \times(0,1), \quad \pi(\xi, E, a)=(E, a),
$$

is proper, i.e., the inverse image through $\pi$ of a compact subset of $\mathcal{E} \times(0,1)$ is compact, too. The proof is complete.

Proof of Proposition 3: By Theorem 1 we know that, for any $E \in \mathcal{E}, t \in \mathbb{N}$ and $a_{t} \in(0,1)$, there exists at least a market equilibrium at time $t$. Let us then check that the equilibrium is unique, omitting as usual the dependence on $t$, in order not to overburden notation. Recalling Definition 2 and the formulation of the market clearing conditions with shares in (3), we have to prove that if the gross substitute property holds for all the individual demand functions there exists one solution $\left(\widehat{p}_{x}, \widehat{p}_{y}\right) \in(0,+\infty)^{2}$ to the system

$$
a x_{\alpha}^{*}\left(p_{x}, p_{y}\right)+(1-a) x_{\beta}^{*}\left(p_{x}, p_{y}\right)-w_{x}=0=a y_{\alpha}^{*}\left(p_{x}, p_{y}\right)+(1-a) y_{\beta}^{*}\left(p_{x}, p_{y}\right)-w_{y} .
$$

Since we can normalize one price, to show the uniqueness of the solution, we may focus on the price vectors $(1, p) \in(0,+\infty)^{2}$, with $p=p_{y} / p_{x}$, Assuming that $a x_{\alpha}^{*}(1, \widehat{p})+(1-$ a) $x_{\beta}^{*}(1, \widehat{p})-w_{x}=0=a y_{\alpha}^{*}(1, \widehat{p})+(1-a) y_{\beta}^{*}(1, \widehat{p})-w_{y}$, let us prove that no $(1, \widetilde{p})$, with $\widetilde{p} \neq \widehat{p}$, may solve (25). Namely, if $\widetilde{p}<\widehat{p}$, by the gross substitute property it would hold that $x_{i}^{*}(1, \widetilde{p})<x_{i}^{*}(1, \widehat{p}), i \in\{\alpha, \beta\}$, and thus, since $a \in(0,1)$, we would have

$$
a x_{\alpha}^{*}(1, \widetilde{p})+(1-a) x_{\beta}^{*}(1, \widetilde{p})-w_{x}<a x_{\alpha}^{*}(1, \widehat{p})+(1-a) x_{\beta}^{*}(1, \widehat{p})-w_{x}=0,
$$

so that $(1, \widetilde{p})$ would not be a solution to $(25)$. Similarly, if $\widetilde{p}>\widehat{p}$, by the gross substitute property it would hold that $x_{i}^{*}(1, \widetilde{p})>x_{i}^{*}(1, \widehat{p}), i \in\{\alpha, \beta\}$, and thus, since $a \in(0,1)$, it would follow that

$$
a x_{\alpha}^{*}(1, \widetilde{p})+(1-a) x_{\beta}^{*}(1, \widetilde{p})-w_{x}>a x_{\alpha}^{*}(1, \widehat{p})+(1-a) x_{\beta}^{*}(1, \widehat{p})-w_{x}=0,
$$

so that $(1, \tilde{p})$ would not solve $(25)$

This completes the proof.

Proof of Proposition 4: The conclusion immediately follows by observing that the solutions to the fixed-point equation $g(a)=a$, with $g$ as in (10), are given by $a=0, a=1$, as well 
as by all solutions to the equation $V_{\alpha}(a)=V_{\beta}(a)$, if any, and by all the solutions to the equation $\left(V_{\alpha}(a)+V_{\beta}(a)\right) / 2=\bar{V}$, if any. Namely, the nontrivial equilibria for equation (9) are found as solutions to the equation $\mathscr{A}_{\alpha}=f\left(\sigma d^{2}\left(\bar{V}, V_{\alpha}\right)\right)=f\left(\sigma d^{2}\left(\bar{V}, V_{\beta}\right)\right)=\mathscr{A}_{\beta}$. Since $f$ is strictly decreasing, all solutions have to satisfy $d\left(\bar{V}, V_{\alpha}\right)=d\left(\bar{V}, V_{\beta}\right)$, i.e., $V_{\alpha}=V_{\beta}$ or $\left(V_{\alpha}+V_{\beta}\right) / 2=\bar{V}$, as desired. This concludes the proof.

Proof of Proposition 5: Since $g^{\prime}(0)=1 /\left(\exp \left(\mu\left(\mathscr{A}_{\beta}(0)-\mathscr{A}_{\alpha}(0)\right)\right)\right)$, the condition $g^{\prime}(0)>$ -1 is always fulfilled, while $g^{\prime}(0)<1$ is fulfilled for $\mathscr{A}_{\beta}(0)>\mathscr{A}_{\alpha}(0)$. Independently of $f$, exploiting the strictly decreasing behavior of $\mathscr{A}_{i}$ with respect to $d^{2}\left(\bar{V}, V_{i}\right)$, we then find $\left(V_{\alpha}(0)-\bar{V}\right)^{2}>\left(V_{\beta}(0)-\bar{V}\right)^{2}$, as desired.

As concerns the local stability of $a=1$ for map $g$, we find that $g^{\prime}(1)=$

$\exp \left(\mu\left(\mathscr{A}_{\beta}(1)-\mathscr{A}_{\alpha}(1)\right)\right)$. Hence, the condition $g^{\prime}(1)>-1$ is always fulfilled, while $g^{\prime}(1)<1$ is fulfilled for $\mathscr{A}_{\beta}(1)<\mathscr{A}_{\alpha}(1)$. Independently of $f$, exploiting again the strictly decreasing behavior of $\mathscr{A}_{i}$ with respect to $d^{2}\left(\bar{V}, V_{i}\right)$, we find $\left(V_{\alpha}(1)-\bar{V}\right)^{2}<\left(V_{\beta}(1)-\bar{V}\right)^{2}$.

In regard to $a=\hat{a}$, recalling that $V_{\alpha}(\hat{a})=V_{\beta}(\hat{a})$, we find that $g^{\prime}(\hat{a})=1-2 \mu \sigma \hat{a}(1-$ $\hat{a})\left.\frac{\partial \mathscr{A}_{\beta}(a)}{\partial\left(\sigma d_{\beta}^{2}\right)}\right|_{a=\hat{a}}\left(V_{\beta}(\hat{a})-\bar{V}\right)\left(V_{\beta}^{\prime}(\hat{a})-V_{\alpha}^{\prime}(\hat{a})\right)$. Since $\left.\frac{\partial \mathscr{A}_{\beta}(a)}{\partial\left(\sigma d_{\beta}^{2}\right)}\right|_{a=\hat{a}}$ is negative, then $a=\hat{a} \in(0,1)$ is unstable for map $g$ for all positive values of $\mu$ if $\left(V_{\beta}(\hat{a})-\bar{V}\right)\left(V_{\beta}^{\prime}(\hat{a})-V_{\alpha}^{\prime}(\hat{a})\right)>0$. If instead $\left(V_{\beta}(\hat{a})-\bar{V}\right)\left(V_{\beta}^{\prime}(\hat{a})-V_{\alpha}^{\prime}(\hat{a})\right)<0$, then $g^{\prime}(\hat{a})<1$ is always fulfilled, while, if $\mathscr{A}_{\beta}$ does not depend on $\mu, g^{\prime}(\hat{a})>-1$ is fulfilled for $\mu<\hat{\mu}$, with $\hat{\mu}$ as in (11), obtaining the desired conclusion about the local stability of $a=\hat{a} \in(0,1)$ for map $g$, too. The condition for the flip bifurcation follows by setting $g^{\prime}(\hat{a})=-1$.

Finally, in regard to $a=\tilde{a}$, recalling that $V_{\beta}(\tilde{a})-\bar{V}=\bar{V}-V_{\alpha}(\tilde{a})$, we find that $g^{\prime}(\tilde{a})=$ $1-\left.2 \mu \sigma \tilde{a}(1-\tilde{a}) \frac{\partial \mathscr{A}_{\beta}(a)}{\partial\left(\sigma d_{\beta}^{2}\right)}\right|_{a=\tilde{a}}\left(\bar{V}-V_{\alpha}(\tilde{a})\right)\left(V_{\alpha}^{\prime}(\tilde{a})+V_{\beta}^{\prime}(\tilde{a})\right)$. Since $\left.\frac{\partial \mathscr{A}_{\beta}(a)}{\partial\left(\sigma d_{\beta}^{2}\right)}\right|_{a=\tilde{a}}$ is negative, then $a=\tilde{a} \in(0,1)$ is unstable for map $g$ for all positive values of $\mu$ if $\left(\bar{V}-V_{\alpha}(\tilde{a})\right)\left(V_{\alpha}^{\prime}(\tilde{a})+V_{\beta}^{\prime}(\tilde{a})\right)>$ 0 . If instead $\left(\bar{V}-V_{\alpha}(\tilde{a})\right)\left(V_{\alpha}^{\prime}(\tilde{a})+V_{\beta}^{\prime}(\tilde{a})\right)<0$, then $g^{\prime}(\tilde{a})<1$ is always fulfilled, while, if $\mathscr{A}_{\beta}$ does not depend on $\mu, g^{\prime}(\tilde{a})>-1$ is fulfilled for $\mu<\tilde{\mu}$, with $\tilde{\mu}$ as in (12). The condition for the flip bifurcation follows by setting $g^{\prime}(\tilde{a})=-1$. This concludes the proof. 